\title{
Expectancies Influence Attention to Neutral But Not Necessarily to Threatening Stimuli: An fMRI Study
}

Running Head: Expectancies and Attention to Threat

Tatjana Aue ${ }^{\mathrm{a}, \mathrm{b}^{*}}$, Raphaël Guex ${ }^{\mathrm{b}, \mathrm{c}}$, Léa A. S. Chauvigné ${ }^{\mathrm{d}}$, Hadas Okon-Singer ${ }^{\mathrm{e}}$, and Patrik Vuilleumier $^{\text {b,c }}$

${ }^{a}$ Department of Psychology, University of Bern, Bern, Switzerland ${ }^{\mathrm{b}}$ Swiss Center for Affective Sciences, University of Geneva, Geneva, Switzerland ${ }^{c}$ Department of Neurology, University Hospitals of Geneva, Geneva, Switzerland ${ }^{\mathrm{d}}$ Department of Psychology, McMaster University, Hamilton, Canada ${ }^{\text {e}}$ Department of Psychology, University of Haifa, Haifa, Israel

Number of figures: 4

Number of tables: 2

\section{*Correspondence:}

Tatjana Aue

University of Bern

Institute of Psychology

Division of Experimental Psychology and Neuropsychology

Fabrikstr. 8

3012 Bern

Switzerland

tatjana.aue@psy.unibe.ch

Phone: +41316314095 


\begin{abstract}
Recent behavioral observations suggest an influence of prior expectancies on attention to neutral targets, while the detection of threatening targets remains comparably immune to these expectancies. The origin of this asymmetry, however, remains unclear. Here, therefore, we investigated its neural basis by using fMRI. Specifically, we tested whether, in accordance with the idea of a resetting attentional system during phylogenetic threat detection, neural responses for threatening compared with neutral targets would remain largely unaffected by prior expectancies. Alternatively, neural responses could reflect equally strong expectancy influences on both types of targets, with the respective patterns differing, thereby producing the asymmetric effect observed in behavior. Predictive cues in our study evoked specific behavioral and neural expectancy states and effectively modulated response latencies to detect neutral (bird) targets in a $3 \times 3$ visual search matrix: When threat-related (spider) rather than neutral targets were expected, bird detection was considerably slowed, and the neural response to expected birds differed from that to unexpected birds. Conversely, and in line with the hypothesis of a resetting attentional system for phylogenetic threat, expectancy cues had no impact on reaction times or neural responses for spider targets - either in spider phobics or in non-spider-fearful controls. Our data support the idea of bottom-up enhancement of threat-related information through processing pathways unaffected by topdown modulatory influences such as expectancy. These pathways may subserve rapid and comparably automatic responding to threat stimuli by safeguarding independence from more controlled and explicit expectancies, consequently promoting adaptive behavior and survival.
\end{abstract}

Keywords: expectancy bias, attention bias, combined bias hypothesis, fear, phobia, biological preparedness, functional magnetic resonance imaging (fMRI) 


\section{Expectancies Influence Attention to Neutral But Not Necessarily to Threatening Stimuli: An fMRI Study}

Fear is pain arising from the anticipation of evil. - Aristotle

As noted by Aristotle, anticipation (i.e., expectancy) of threat is a powerful determinant of fear. Such a view receives support by the observation of so-called expectancy biases that are considered to be a hallmark of anxiety disorders (e.g., animal phobia, social phobia, and flight phobia; Aue \& Hoeppli, 2012; Foa \& Kozak, 1986; Mühlberger, Wiedemann, Hermann, \& Pauli, 2006; Muris, Huijding, Mayer, den Breejen, \& Makkelie, 2007). For example, even when they receive background information about the objective likelihood of encountering different animals, spider phobics (but not non-spider-fearful controls) systematically overestimate the likelihood of encountering spiders rather than snakes or birds (Aue \& Hoeppli, 2012; Aue, Hoeppli, Piguet, Hofstetter, Rieger, \& Vuilleumier, 2015). Recently, these biased expectancies in spider phobics have been shown to involve deactivations in key integration and regulation centers in the brain, such as the precuneus and the lateral prefrontal cortex; the more strongly these areas are deactivated, the stronger the displayed bias is (Aue et al., 2015).

While these findings are compelling, the exact functions of anticipation for threatrelated behavior preparation remain to be determined. It seems plausible to assume that expectancy of threat enhances visual attention, thus permitting successful detection of the threat source in the environment. Such a process could hence increase the chance of adaptively responding to a threatening situation (cf. Mohanty \& Sussman, 2013). In line with the hypothesis of such an association of threat-related expectancies and attention enhancement, highly fearful and phobic individuals are characterized by both expectancy 
biases (e.g., exaggerated expectancies of encountering a feared threat) and deviated attentional processes (see Aue \& Okon-Singer, 2015, for a detailed overview). Apart from those for expectancy biases, neural substrates have also been identified for attention biases: The amygdala is proposed to be the key structure implicated in early automatic vigilance for threat, whereas the prefrontal cortex is thought to mediate more controlled biased attentional responding, namely, difficulty in disengaging attention from threat once it has been oriented to and attentional avoidance of threat (see Cisler \& Koster, 2010, for a division of attention biases into three distinct categories).

A paradigm that has often been used to study attention bias in animal phobia is the visual search task (Flykt, Lindeberg, \& Derakshan, 2012; Öhman, Flykt, \& Esteves, 2001; Soares, Esteves, Lundqvist, \& Öhman, 2009). In the simplest version of this task, a threatening target appears among neutral objects or a neutral target appears among threatening stimuli. The participant's task is to detect the deviant stimulus as quickly as possible. Shorter reaction times (RTs) to threatening compared with neutral targets reflect vigilance to threat. Interestingly, the effect can be observed in both phobics and non-fearful individuals, suggesting that human beings are biologically prepared for the detection of phylogenetic threat (cf. Öhman \& Mineka, 2001; Seligman, 1971).

Indeed, a superefficient information processing system for threat may be at the basis of several maladaptive information processing biases (e.g., related to attention, interpretation, expectancies, and memory) that are thought to provoke and maintain pathological fear and anxiety (e.g., Aue \& Okon-Singer, 2015; Butler \& Mathews, 1983; Hirsch, Clark, \& Mathews, 2006; Mathews, Mackintosh, \& Fulcher, 1997; Taylor \& Rachman, 1994). Notably, the existence of such biases in healthy individuals has not been consistently observed (e.g., Bar-Haim, Lamy, Pergamin, Bakermans-Kranenburg, \& van IJzendorn, 2007). 
Recent theoretical accounts state that different types of information processing biases in pathological fear and anxiety (as in depression) mutually influence and stabilize or even enhance each other (e.g., Aue \& Okon-Singer, 2015; Hirsch et al., 2006; Peschard, \& Philippot, 2015; see also Everaert, Koster, \& Derakshan, 2012; Ingram, 1984; J. M. G. Williams, Watts, MacLeod, \& Mathews, 1997). In fact, there is evidence that the amount of time that individuals spend gazing on images of spiders (a global measure of visual attention) is related to their expectancies of encountering these animals (Aue, Hoeppli, Piguet, Sterpenich, \& Vuilleumier, 2013). Moreover, the nature of this association may be an indicator of one's state of health: In phobic individuals, we found that shifts toward visual avoidance of threatening animals were accompanied by reduced expectancies of encountering these animals, on the one hand, and by reduced physiological arousal and reduced activity in the amygdala, orbitofrontal cortex (OFC), anterior cingulate cortex (ACC), and precuneus, on the other hand. Conversely, non-fearful controls displayed a positive association between attention engagement and encounter expectancies, as well as physiological arousal and neural activity in the aforementioned brain regions. Together, these findings suggest that visual avoidance in spider phobia may serve to downregulate threat expectancies and the associated negative bodily consequences, whereas healthy individuals more efficiently regulate their affective state by paying greater attention to potential threat sources.

However, it is yet unclear whether the links between threat-related expectancies and attention are causal or even bidirectional. While it is intuitive to speculate that threat anticipation determines attention deployment, it is not necessarily true. At least two visual search studies (Aue, Chauvigné, Bristle, Okon-Singer, \& Guex, 2016; Aue, Guex, Chauvigné, \& Okon-Singer, 2013) revealed that expectancy may influence visual attention to neutral but not inevitably to threatening objects. Specifically, in these two experiments, spider-phobic and non-spider-fearful control participants were preinformed in each experimental trial about 
the likelihood of two different animals (threatening spider vs. neutral bird) being the target in a subsequently presented $3 \times 3$ search matrix that contained eight butterflies as distractors. In some trials, the likelihood of a spider being the target was announced to be much higher than the likelihood of a neutral bird being the target; in other trials, it was the reverse; and in still other trials, participants were informed that there was an equal probability of either being the target.

In neither study did expectancies regarding the apparition of the threat source (i.e., spider) significantly impact its detection (i.e., the vigilance to it). Interestingly, the absence of statistically significant expectancy influences on threat-related attention was not population specific, as it held for both spider-phobic and control participants, the latter having claimed to not fear spiders at all. Notably though, the same expectancies (provoked by the exact same expectancy cues) did influence attention to neutral bird targets in both populations. Moreover, this asymmetric association between expectancies and attention deployment was also shown by means of physiological measures (Aue et al., 2016). Although, apart from error rates and RTs, we found a robust indication for expectancy influences on attention to birds in pupil diameter and heart rate, the same expectancies did not significantly impact attention to spiders; only heart rate displayed a weak and transient effect of prior expectancies on the orientation of attention to threat. Together, these findings point to the possibility that expectancy-attention links fundamentally differ between threat-related and neutral targets.

The influence of the exact same predictive cues on bird but not spider detection is intriguing. In fact, we had initially anticipated that encounter expectancy for spiders (i.e., when the likelihood of a spider target was announced to be much higher than the likelihood of a neutral bird target) would create a mental template of a spider that might then facilitate the detection of a spider (cf. predictive coding theory; Summerfield et al., 2006; Zelano, Mohanty, \& Gottfried, 2011; for further evidence of memory-guided attention, see Duncan \& 
Humphreys, 1989; Hutchinson \& Turk-Browne, 2012; Wolfe, 2010; Wolfe, Butcher, Lee, \& Hyle, 2003). Facilitated detection following specific types of anticipation could then be a result of pattern matching - especially so for threatening material because, in that case, the encounter expectancy would also signal urgency. That our statistical analyses, which were inconsistent with such a point of view and opposed to findings for neutral targets, did not reveal significant expectancy effects for threatening targets speaks to highly specified and efficient detection mechanisms for threat that may function independently of momentary expectancy states.

To yield better insights, in the current paper, we investigated the neural basis of this known expectancy-attention asymmetry in the behavioral domain. Notably, while such an examination may seem mostly replicative at first sight, the present investigation has a much greater impact. This is because knowledge about brain functioning can substantially increase our understanding of overt behavior. In an ideal case, assessment of neural activity patterns helps identify the origin and function of behavioral phenomena (Aue, Lavelle, \& Cacioppo, 2009). For instance, even if there is no behavioral difference observed between experimental conditions, neuroimaging data can still point to diverging underlying mechanisms (e.g., Beauchamp, Kahn, \& Berkman, 2016; Cohen et al., 2016).

With respect to our own research, at least three different hypotheses can be contrasted and informed by neuroimaging methods. First, it could be hypothesized that expectancy confirmation and violation for neutral and phylogenetic threat targets are processed similarly in the early processing stream, with unexpected targets needing greater attention than expected targets. The RT asymmetry described in our previous publications (Aue, Chauvigné, Bristle, Okon-Singer, \& Guex, 2016; Aue, Guex, Chauvigné, \& Okon-Singer, 2013) could then arise later on in the motor preparation phase. In that case, one would predict expectancyrelated differences in attention to threat versus neutral targets to be reflected in motor activity. 
That we did not see differences in RTs between expected and unexpected spiders-despite the possibility that these might be processed differently in the early processing stream-could result from the existence of emergency (i.e., compensation) mechanisms that provide boosted response preparation in the case of false (and potentially harmful) safety expectations.

Second, differences between the targets may already arise in the early processing stream. While effects related to expectancy violation and confirmation might exist for both types of targets, they could be qualitatively different for threatening versus neutral targets (i.e., associated with different neural responding). The neural substrates concerned could include, among others, the visual cortex. In sum, both hypotheses would imply the existence of clearcut neural differences between unexpected and expected spiders.

On the other hand, attentional mechanisms have often been suggested to substantially differ for neutral versus phylogenetic threat stimuli (e.g., Öhman \& Mineka, 2001; Seligman, 1971), with the processing of the latter being characterized by highly automatized preattentive processing. Correspondingly, in earlier publications on the topic (Aue, Chauvigné, Bristle, Okon-Singer, \& Guex, 2016; Aue, Guex, Chauvigné, \& Okon-Singer, 2013), we posited a third possibility, namely, that expectancy states often cannot successfully penetrate the attention system responsible for the detection of phylogenetic threat. To ensure that necessary available resources are fully attributed to an existing threat in the environment, attention deployment during rapid responding to threat could be largely shielded from top-down processes such as prior induced expectancies. In line with the idea of preattentive processing, there could instead be neural resetting whenever such a threat is presented. If this were true, we would not find any or only a small difference in brain activations during the detection of expected versus unexpected threat (while, at the same time, pronounced differences could arise for neutral stimuli to which other attentional mechanisms would apply). According to such a viewpoint, even early perceptual processing of the threatening target would be 
uninfluenced by prior presented expectancy cues; neural resetting would prevent the organism from lingering in false beliefs of security and ensure rapid responding in emergency situations.

In line with this latter view, in the current study, we hypothesized that anticipation has only a negligible impact on neural responding related to attention deployment (and response preparation) for threatening objects, whereas it clearly affects neural responding related to attention deployment (and response preparation) for neutral objects. To this aim, we used the same experimental task with spider-phobic and control participants as we did previously (Aue et al., 2016; Aue, Guex et al., 2013), but now probed for functional brain responses (as assessed by magnetic resonance imaging [MRI]) that arise during task performance.

In accordance with our previous RT and peripheral physiology findings, we first hypothesized that brain responses during target detection would display clear activity differences between expected and unexpected birds. Specifically, we predicted increased activity for unexpected compared with expected birds in frontoparietal and occipital sites that have often been reported in research on top-down control of visual attention and visual selection (e.g., Corbetta, 1998; Rolls, 2013; Soto, Humphreys, \& Rotshtein, 2007). By contrast, similar effects were not anticipated for expected versus unexpected spiders.

Second, we predicted neural responses that are characteristic of the expectancy state during cue presentation (i.e., degree of cue discrimination; expected target is a bird vs. a spider) to determine RTs to bird targets but not RTs to spider targets in the proceeding detection phase. This latter hypothesis thus expresses our conviction that those individuals whose neural activity most strongly differentiated spider from bird cues were those who were mostly influenced by the cues and thus subsequently showed greatest slowing of RTs to unexpected compared with expected birds. A resetting attention system for threat, in contrast, 
would not be influenced by prior expectancies (i.e., cue distinctions); that is why we did not postulate a comparable correlation for the threatening spider targets.

\section{Method}

\section{Participants}

Forty-five participants (21 spider phobic; 10 male [four in the spider-phobic and six in the control group]), aged between 18 and 34 years $(M=23.5, S D=4.10)$ were recruited via ads placed in university buildings, as well as on university and local websites. The study was embedded in a larger project investigating decision making and psychophysiological responses during the imagination of encounters with feared and non-feared animals. The ads explicitly specified these project aims. Persons interested in the study were interviewed by telephone and checked for criteria of the Diagnostic and Statistical Manual of Mental Disorders (4th ed., text rev.; DSM-IV-TR; American Psychiatric Association, 2000) and the International Statistical Classification of Diseases and Related Health Problems (10th rev.; ICD-10; World Health Organization; 1992) for the presence or absence of spider phobia (adapted from Mühlberger et al., 2006). Participants were included in the study if they (a) met the $D S M-I V-T R$ and $I C D-10$ criteria for spider phobia (spider-phobic group), or (b) fulfilled none of the criteria and additionally claimed to not fear spiders (control group). Another condition for inclusion in the study was a body mass index between 18.5 and 25 . Exclusion criteria comprised neurological, cardiovascular, respiratory, and psychiatric diseases other than spider phobia, as well as medication use, pregnancy, claustrophobia, and metal implants.

Fear of spiders was further assessed after the experiment with the French translation of the Fear of Spiders Questionnaire (Szymanski \& O’Donohue, 1995), t(43) =14.40, $p<$ $.000001(M \mathrm{~s}=89.6$ and 28.9, $S D \mathrm{~s}=15.05$ and 13.20, for phobic and control groups, respectively). Participants in the two groups did not differ with respect to age, $t(43)=-0.93$, $n s(M \mathrm{~s}=22.9$ and 24.0 years, $S D \mathrm{~s}=3.61$ and 4.50 years, for phobic and control groups, 
respectively); trait anxiety, $t(43)=0.67, n s(M s=40.9$ and $39.1, S D s=8.31$ and 8.93 , for phobic and control groups, respectively); and state anxiety, $t(42)=1.25, n s(M \mathrm{~s}=35.3$ and $31.9, S D s=9.45$ and 10.23 , for phobic and control groups, respectively; due to time pressure, one participant in the control group did not complete this scale), as measured with the French version (Gauthier \& Bouchard, 1993) of the State-Trait Anxiety Inventory (original English version: Spielberger, 1983).

\section{Stimuli and Experimental Paradigm}

The experimental task was a visual search task similar to that in Aue, Guex, et al. (2013) and included the presentation of three types of expectancy cues that were thought to facilitate or inhibit the detection of spider and bird targets in a search array (see Procedure for further details).

Cues (expectancy manipulation). Three types of verbal cues were presented as text on the computer screen. The first, "spider $90 \%$," specified a $90 \%$ probability that the to-bedetected (deviant) target in the subsequently presented search array would be a spider among eight butterflies. Because the target could have been either a spider or a bird, this cue expressed a high probability for a spider target and a low probability for a bird target. The second cue, "bird 90\%," specified a 90\% probability that the to-be-detected target in the subsequently presented search array would be a bird. Thus, this cue expressed a high probability for a bird target and a low probability for a spider target. Finally, the third cue, "spider bird 50\%" (for half of the participants; for the other half, the third cue was "bird spider 50\%"), specified an equal probability for a spider or a bird to be the target in the search array. However, 19 (10 phobics) of 39 participants mentioned after completion of the experiment that they had nonetheless believed spiders to appear more frequently than birds in these ambiguously cued trials. Another three phobic participants mentioned having expected 
bird targets to appear more frequently than spider targets in the ambiguous trials.

Consequently, we did not include this condition in the current analyses (corresponding effects, however, corroborate our interpretation of the data from the unambiguous trials and can be found in the Supplementary Materials, section C: addition to Step 3 analyses).

In reality, the spider 90\% [bird 90\%] cue (for simplicity, termed spider [bird] cue in the following) condition referred to a probability of $70 \%$ that there would actually be a spider [bird] target among eight butterflies in the search array presented thereafter. This discrepancy occurred because we had aimed at reducing the duration of the experiment while keeping the cues highly salient and ensuring enough trials in each experimental condition; refer to the Limitations section for details. In the remaining cases, either a bird [spider] target was presented or no deviant at all. The latter trials were included to verify that the participants responded on the basis of target perception. In the 50\% (i.e., ambiguous, unconsidered) cue condition, in contrast, there was indeed an equal likelihood of either a spider or a bird being the target in the subsequently shown search array.

In sum, the total number of 244 experimental trials consisted of 136 match trials (68 spider cue-spider target trials and 68 bird cue-bird target trials), 48 mismatch trials ( 24 spider cue-bird target trials and 24 bird cue-spider target trials), 48 ambiguously cued trials (24 ambiguous cue-spider target trials and 24 ambiguous cue-bird target trials), and 12 no-target trials (four trials for each spider cue, bird cue, and ambiguous cue).

Visual search array (used to measure attention). In each trial, the search array consisted of a matrix of nine different animal pictures with three columns and three rows. There was no space in-between the pictures, and each picture within the matrix was displayed at $341 \times 256$ pixels. Stimuli consisted of (a) 30 pictures displaying spiders, all taken from the Geneva Affective PicturE Database (GAPED; Dan Glauser \& Scherer, 2011); (b) 30 birds, 
collected from the Internet; and (c) 100 butterflies, also collected from the Internet. The animals covered virtually the whole picture (see example of a search array in Figure 1).

The butterfly distracters were selected based on the assumption that they share significant features with both spiders and birds (e.g., wings corresponding to birds' wings; six legs and two antennas corresponding to the eight legs of spiders). Seventy-five percent of the butterflies were displayed from their side, clearly showing all legs and antennas. All images were displayed in gray scale, preventing pop-out effects based on color differences between stimulus categories. The stimuli in the two categories were matched for luminance and contrast, and spider and bird targets appeared with an equal probability in any of the nine different locations within the matrix.

Insert Figure 1 about here

\section{Procedure}

Upon participants' arrival at the laboratory, the nature of the experiment was explained and written informed consent was obtained (protocol approved by the local ethics committee and in accordance with the Helsinki Declaration of Human Rights; World Medical Association, 1999). The experimental task was introduced as a test of the capacity to detect spiders and birds in an array of butterflies and performed in an MRI scanner. After participants had thoroughly read the instructions for the task, they performed 10 practice trials to become familiar with the task. If they had no questions, they were comfortably positioned in an MRI scanner. 
In each experimental trial, participants saw a fixation cross that was followed by a cue. The cue referred to the probability that the to-be-detected target in the subsequently presented search array would be a spider or a bird (see preceding section for details). After the presentation of the cue, another fixation cross appeared. Next, the search array consisting of nine pictures (either nine butterflies [no target], eight butterflies and a spider, or eight butterflies and a bird) was shown and the participants had to decide whether there was no target, or whether the target was a spider or a bird.

Participants were instructed to react as fast and as correctly as possible. Responses were given by pressing three different buttons of a response button box (counterbalanced across participants). In total, 244 experimental trials were presented in random order in four runs of 61 trials with short pauses in between. The average intertrial interval was $9.5 \mathrm{~s}$ (range: 9-10 s; for an example of an experimental trial [task sequence], see Figure 1). Jitters included between cues and targets permitted statistical separation of blood oxygenation leveldependent responses for cues and targets. To additionally disentangle cue from target processing, we included 5\% no-target trials. After the task, an anatomical scan of the participants' brain was acquired. Outside the scanner, the participants then completed the Fear of Spiders Questionnaire and the trait and state scales of the State-Trait Anxiety Inventory before they were debriefed.

\section{Functional MRI (fMRI) Data Acquisition and Image Processing}

Functional images were acquired by using a 3T whole-body MRI scanner (Trio TIM, Siemens) with a 32-channel head coil. We used a multiplexed EPI sequence (Feinberg et al., 2010), allowing for a rapid temporal resolution with $\mathrm{TR}=650 \mathrm{~ms}, \mathrm{TE}=30 \mathrm{~ms}$, flip angle $=$ $50^{\circ}, 36$ slices, $64 \times 64$ pixels, $3 \times 3 \times 3 \mathrm{~mm}^{3}$ voxel size, and $3.9 \mathrm{~mm}$ slice spacing. The multiband acceleration factor was 4, and a parallel acquisition technique (PAT) was not used. Structural images were acquired with a T1-weighted 3D sequence (MPRAGE, TR/TI/TE = 
1900/900/2.27 ms, flip angle $=9^{\circ}$, PAT factor $=2$, voxel dimensions: $1 \mathrm{~mm}$ isotropic, $256 \times$ $256 \times 192$ voxels). An automatic shimming procedure was performed to minimize inhomogeneities of the static magnetic field. At the beginning of each session, image acquisition started after the recording of three dummy volumes to avoid T1 saturation effects. Participants wore earplugs to attenuate scanner noise; head movement was restricted by a vacuum pillow.

MRI data were preprocessed and analyzed with SPM8 (Wellcome Dept. of Imaging Neuroscience, London, UK; http://www.fil.ion.ucl.ac.uk/spm). Functional images were spatially realigned to the first volume by rigid body transformation, spatially normalized to the standard Montreal Neurological Institute EPI template to permit group analysis, and spatially smoothed with an isotropic 8-mm full-width at half-maximum (FWHM) Gaussian kernel (Friston et al., 1995).

\section{Dependent Variables}

Behavioral Data. The dependent behavioral variable consisted of the participants' RTs (in ms) for correct responses in the different experimental conditions of the visual search task.

fMRI Data. We analyzed percentage signal changes in a whole-brain analysis (see subsequent section for details).

\section{Data Analysis}

Exclusion of cases. Four participants (one phobic, three control) made too many errors in the RT task, thus provoking a number of missing values that exceeded one third of all responses in at least one experimental condition (conditions here being defined by the 
combination of the two within-subjects factors Expectancy Cue [spider, bird] and Target [spider, bird] included in our analyses; note that ambiguous cues were not considered because for these cues, most of our participants did not believe in an equal chance of a spider and a bird being the deviant target in the visual search array; for details, refer to the Stimuli and Experimental Paradigm section). In order to ensure a sufficiently high number of valid trials, we removed the data of these four participants altogether. The data of two additional control participants were removed because the first had been accidentally scanned with a different sequence and the second provoked too many movement artifacts. Errors in the experimental task of the remaining participants (20 phobic, 19 control) made up $\sim 5.8 \%$ of all responses (SD $=3.2 \%$ ), and the corresponding data were also excluded from RT and fMRI analyses.

Analysis of behavioral data. A $2 \times 2 \times 2$ mixed-factorial design resulting from the manipulation of the between-participants factor Population (spider phobic, control) and the within-participants factors Expectancy Cue (spider [spider 90\%], bird [bird 90\%]) and Target (spider, bird) was performed on the participants' RTs (correct responses only). Significant effects as revealed in the performed analysis of variance (ANOVA) were further investigated by the use of post-hoc Tukey tests. An $\alpha$ level of .05 (two-tailed) was applied. All reported effect sizes are partial $\eta^{2}$ and are simply noted as $\eta^{2}$. Because exclusion of outliers (deviating more than 3 SDs from an individual's average RT; 1\% of all responses) yielded similar results, analyses are described for the original data only.

Analysis of fMRI data. We performed standard analyses by using the general linear model as implemented in SPM8. Event-related signal changes were modeled separately for each participant. For each block of the experimental task, we specified a linear model that included 11 event types, corresponding to (a) expectancy phase: presentation of three different cues (spider cue, bird cue, ambiguous cue), and (b) detection phase: the six cue-target combinations (spider cue-spider target, spider cue-bird target, bird cue-spider target, bird 
cue-bird target, ambiguous cue-spider target, ambiguous cue-bird target), as well as no-target trials and those trials in which participants gave incorrect behavioral responses. The length of all events was set to 0 because the perceptual processes that are supposed to happen during these events have a clear onset. Our multiplexed acquisition sequence with short TR (650 ms) allowed us to reliably distinguish different event types despite their rapid succession.

A constant covariate was also added representing the session-specific mean over scans, as well as six motion correction parameters extracted from the realignment procedure. The model included a high-pass filter of $128 \mathrm{~s}$ to remove low-frequency drift of the scanner and first-order auto regressive corrections for auto-correlation between scans. Effects at each brain voxel were estimated by using a least squares algorithm.

Data were analyzed in four steps. First, we investigated neural responses on presentation of the different cues (Step 1: manipulation check; see Supplementary Materials) to verify whether the induction of expectancies in our participants had been successful. Second, we examined whether neural activity differed between the two targets (Step 2: manipulation check; see Supplementary Materials) to test whether these were processed differently. Third, we investigated our major research question, namely, whether, during visual search, expectancies differentially modulated responses to bird but not to or less to spider targets (Step 3). Related to this issue, we further examined whether neural activity in the expectancy phase could successfully predict RTs to bird but not to spider targets in the visual search task (Step 4). Analyses related to the latter two steps are detailed below and were conducted as follows.

\section{Step 3: Differences in neural activity between unexpected and expected (i.e.,} differentially cued) targets. According to our hypothesis of different attentional mechanisms applying to the detection of phylogenetic threat versus neutral stimuli, with a resetting attentional system in case of spider detections, we hypothesized expectancy effects to be 
clearly visible in neural responses to bird targets but not in those to spider targets. To test this hypothesis, we performed a whole-brain analysis in which we first contrasted responses to spider targets that were preceded by bird cues relative to responses to spider targets that were preceded by spider cues (both bird cue $>$ spider cue and spider cue $>$ bird cue; performed on spider targets only). Two different versions of these contrasts were calculated: (a) for both populations together and (b) for spider phobics versus controls (interaction contrast), permitting the identification of spider phobics' particularities in initial responses toward incongruently cued spider targets. Thus, the latter interaction contrast tested whether the differences revealed by the two contrasts specified above (bird cue $>$ spider cue and spider cue > bird cue, performed on spider targets only) were statistically different between phobics and controls (phobics > controls; note that testing the opposite direction was not necessary because the cue contrasts were already tested in both directions). If there was a significant difference, its nature was identified by looking separately into phobics and controls. The same contrasts were performed for the bird targets: bird targets preceded by spider cues versus bird targets preceded by bird cues (also both directions; version a: both populations; version b: contrasting the two populations).

To avoid alpha inflation, we report only significant clusters containing at least 26 contiguous voxels at $p<.001$. This critical cluster size was calculated by a Monte Carlo simulation with 10,000 iterations, assuming some interdependence between voxels (8-mm FWHM), resulting in a corrected whole-brain $p$-value of .001. All parametric maps were rendered on sections of the average $T_{1}$-weighted template brain of all participants.

Step 4: Prediction of RTs for the detection of unexpected versus expected targets by neural activity for spider versus bird cues in the expectancy phase. We hypothesized that selective modulation of brain activity by the expectancy cues would affect the detection of birds but not the detection of spiders in the subsequent visual search task. The latter relates to 
our conviction that participants whose neural activity most strongly differentiated between spider and bird cues were those who were mostly influenced by the cues and thus subsequently showed greatest slowing of RTs to unexpected compared with expected birds. A resetting attentional system for phylogenetic threat, in contrast, would prohibit such an effect from being seen in the RTs for spider targets. To examine this hypothesis, we adopted a whole-brain between-participants approach and correlated participants' neural responses to spider versus bird cues (spider cue-bird cue) in the expectancy phase with the participants' RTs for unexpected versus expected spiders (RT unexpected-RT expected); we tested for both a positive and a negative association. Similarly, we correlated participants' neural responses to bird versus spider cues (bird cue-spider cue) in the expectancy phase with the participants' RTs for unexpected versus expected birds (RT unexpected-RT expected). Comparable analyses as described for Step 3 were calculated. Concretely, we performed both a merged analysis for both populations (version a) and a contrast of the two populations (phobics-controls; version b), the latter analysis relating to a comparison of the size of the observed correlations between phobics and controls for each voxel. In the case of significant version b effects, we additionally performed separate analyses for phobics and controls. Again, a cluster threshold of 26 contiguous voxels at $p<.001$ was applied.

\section{Results}

\section{Behavioral Data}

The 2 (Population: spider phobic, control) $\times 2$ (Expectancy Cue: spider, bird $) \times 2$ (Target: spider, bird) ANOVA on participants' RTs yielded a significant main effect of Population, $F(1,37)=9.59, p<.005, \eta^{2} p=.21$. Overall, phobics displayed shorter RTs than did controls $(M \mathrm{~s}=1053.8$ and $1181.9 \mathrm{~ms}$ for spider phobics and controls, respectively). The ANOVA further yielded a significant main effect of Expectancy Cue, $F(1,37)=49.42, p<$ $.0000001, \eta^{2}=.57$, because of faster responses following bird cues overall $(M \mathrm{~s}=1162.6$ and 
$1073.1 \mathrm{~ms}$ for spider cue and bird cue, respectively). There was also a significant main effect of Target, $F(1,37)=216.22, p<.0000001, \eta^{2}{ }_{p}=.85(M \mathrm{~s}=986.5$ and $1249.2 \mathrm{~ms}$ for spider target and bird target, respectively; Figure 2), corresponding to a global attention bias toward the spider targets.

Both of the latter effects were qualified by the significant interaction Expectancy Cue $\times$ Target, $F(1,37)=16.46, p<.0005, \eta_{p}^{2}=.31$, resulting from the fact that expectancy effects were limited to bird targets. Post hoc Tukey tests for this interaction showed no difference between the expectancy cues for spider targets $(p=.23 ; M \mathrm{~s}=1004.3$ and $968.8 \mathrm{~ms}$ for spider cue and bird cue, respectively), whereas RTs were longer for those bird targets that had been preceded by spider cues rather than bird cues (i.e., that were unexpected rather than expected; $p=.0002 ; M \mathrm{~s}=1320.8$ and $1177.5 \mathrm{~ms}$ for spider cue and bird cue, respectively).

Finally, the significant interaction Population $\times$ Target, $F(1,37)=33.28, p<$ $.0000005, \eta^{2}=.47$, arose because phobics and controls did not display different RTs for the bird targets (Tukey test for this pairwise comparison: $p=0.94 ; M \mathrm{~s}=1236.7$ and $1261.7 \mathrm{~ms}$ for phobics and controls, respectively), but phobics were faster at detecting spiders than were controls $(p=.0002 ; M \mathrm{~s}=871.0$ and $1102.1 \mathrm{~ms}$ for phobics and controls, respectively). Both groups were characterized by shorter RTs for spider compared with bird targets (both $p$ s $=$ .0002 ; i.e., attention bias for threat). Neither the interaction Population $\times$ Expectancy Cue nor the interaction Population $\times$ Expectancy Cue $\times$ Target reached significance, $F \mathrm{~s}(1,37)=0.24$ and $2.29, p s=.63$ and $.14, \eta^{2}{ }_{s}=.01$ and 06 , respectively. 


\section{fMRI Data}

In the following, we describe fMRI data related to the test of our hypothesis that expectancies successfully influence attention deployment for bird targets but—due to a resetting attentional system for threat detection — the same is not the case for spider targets. Results regarding neural activity for spider versus bird cues in the expectancy phase (without consideration of the later-presented targets) are presented in the Supplementary Materials and Table S1 (Step 1); results regarding neural activity for spider versus bird targets in the detection phase (without consideration of the previously presented cues) are presented in the Supplementary Materials and Table S2 (Step 2).

\section{Differences in neural activity between unexpected and expected targets (Step 3).}

First, we compared the neural activations related to unexpected spiders and expected spiders during spider detection in the visual search task. To this aim, we calculated both the contrast [spider target preceded by bird cue] - [spider target preceded by spider cue] and the same contrast in the opposite direction [spider target preceded by spider cue] - [spider target preceded by bird cue]. In line with our predictions, there were no significant effects: Neither was there a significant difference in neural responding for unexpected versus expected spiders (both directions of the above specified contrasts) when both populations were analyzed together (version a; see Method section for details), nor were these contrasts any different between the two populations (interaction contrast; i.e., version b).

Instead, in agreement with our RT data, our brain data revealed a robust influence of expectancies on bird detection (Table 1, Figure 3). The combined analysis of both populations (version a) demonstrated that expectancies evoked by the preceding cues indeed powerfully influenced brain responses to the bird targets. Most notably, unexpected birds compared with expected birds produced greater activity in the inferior frontal gyrus in both spider phobics and controls. In addition, our participants demonstrated increased activity in various 
dorsolateral and dorsomedial prefrontal areas, as well as in the superior and inferior parietal lobule. Finally, contrasting the responses of the two populations (interaction contrast; i.e., version b) did not yield any significant difference.

Together, these results support the notion that the detection of threat-related stimuli did not or only marginally engaged different brain areas when expected or unexpected, whereas the detection of unexpected neutral stimuli, in line with our hypotheses, activated areas involved in attention control and task switching. Moreover, results observed were independent of fear level (i.e., population).

Insert Figure 3 about here

\section{Prediction of RTs for unexpected versus expected targets by neural activity for}

spider versus bird cues in the expectancy phase (Step 4). We hypothesized that participants who most strongly differentiated between the expectancy cues during presentation of the cues would be those who showed a stronger influence of these cues on their later RTs for bird targets. Because of the assumed resetting nature of attentional mechanisms characteristic of the detection of phylogenetic threat, no such association was predicted for the spider targets. To investigate whether RTs for unexpected versus expected targets could be predicted by cuespecific brain activity in the expectancy phase, we took the fMRI response to bird cue versus spider cue in the expectancy phase and correlated it with RTs for unexpected versus expected targets in the detection phase (refer to Method section for further details).

Notably, analyses performed for spider targets did not yield any significant activation clusters, further underscoring that expectancy influences on attention deployment to spiders was negligible in our experiment. By contrast, for bird targets, activity in the expectancy 
phase significantly predicted the facilitation effect of the expectancy cues on detection speed (Table 2; Figure 4). The whole-brain parametric analysis across both populations (version a) showed that this facilitation effect by the expectancy cues on RTs was associated with selectively increased activity in the precuneus (Figure 4a). Participants who displayed greater precuneus activity for bird compared with spider cues in the expectancy phase were those who showed the greatest increase in RTs for unexpected compared with expected bird targets.

Insert Figure 4 about here

Contrasts of the correlations between the two populations (version b) yielded a significant difference within the posterior cingulate cortex (PCC; Figure 4b). Whereas phobics displayed a negative correlation between PCC activity related to bird-spider cues in the expectancy phase and the facilitation effect expressed in the RTs for bird targets, controls showed a positive correlation.

\section{Discussion}

In the current paper, we describe a visual search task and resulting RT and fMRI data that clearly demonstrate asymmetric effects of expectancies on the detection of neutral (bird) compared with threat-related (spider) targets. Longer RTs for unexpected compared with expected birds indicate that bird detection was strongly influenced by the predictive cues presented prior to search. Detection of spiders, in contrast, was unaffected by the preceding cues. Results are thus highly consistent with previous studies (that were based on the same experimental paradigm) in which RTs (Aue et al., 2013) and autonomic nervous system measures were used (Aue et al., 2016). 
More important, here we additionally delineated the neural basis underlying this differential link between expectancy and attention when participants searched for phylogenetic threat versus neutral information. We had hypothesized the existence of resetting attentional mechanisms for threat detection that function to safeguard survival in emergency situations. Greater resistance to expectancy would ensure adequate responding to threats even when observers are unprepared. Whether prior expectancies are confirmed or not should be less relevant in threat-related emergency situations. This issue may instead be processed somewhat independently later on, when the observer is safe again and has sufficient time for it.

Accumulating evidence supports such a point of view: Neural responses as well as RTs and peripheral physiological responses in both the current and earlier investigations (Aue, Guex et al., 2013; Aue et al., 2016) are consistent with it. Specifically, during target detection, coherent neural differences between the expectancy cues were observed only for bird targets in both populations (Step 3): Increased brain activity in various frontal and parietal regions in the detection phase, as well as longer RTs for unexpected compared with expected birds, reflects an impressive influence of these cues on processing and overt behavior. By contrast, comparable differences were not found between unexpected and expected spiders.

Similarly, our additional parametric analysis (Step 4) demonstrated that response facilitation by the cues was restricted to the detection of bird targets. This facilitation was predicted by neural activity in the precuneus and PCC during the expectancy phase. Participants who were characterized by stronger precuneus activity for bird compared with spider cues in the expectancy phase were those who showed the largest increase in RTs to unexpected compared with expected bird targets (PCC results are discussed in more detail later). By contrast and consistent with the idea of a resetting attention system, such 
expectancy-related neural activity had no predictive value for RTs to spider targets. Notably, the same neural activity that effectively predicted the RTs for bird targets did not predict RTs for spider targets. Therefore, the observed asymmetry in expectancy influences on the processing of the two different targets cannot be explained by possible differences in cue effectiveness.

Along these lines, we found clearly distinct neural response patterns associated with the expectancy cues across target conditions (see Supplementary Materials, Step 1, for details), especially in the phobics. This observation underscores once more that the cues had been effective and had a high significance for these participants. Our findings hence highlight that recognition of different likelihoods for the apparition of spider targets does not suffice to drive attention orientation toward or away from threatening spider targets. Instead, these data favor the idea that early automatic attention deployment to threat (as revealed in the participants' RTs) — as opposed to attention deployment to neutral events — is indeed more independent from prior expectancy states.

A review of the literature performed by Rolls (2013) suggests that attention and expectancies recruit a common neural network: Both cognition (encompassing expectancies) and attention can exert top-down influences on the processing of bottom-up sensory inputs, and this influence is mediated in part by the ACC and the OFC. Such top-down influences could promote the activation of selective neuronal assemblies in the early information processing stream while simultaneously inhibiting others. Hence, specific stimulus representations will be enhanced and others suppressed (e.g., Desimone \& Duncan, 1995), so that subsequent processing of incoming information will be biased in favor of currently relevant content.

Consistent with this view, in the present study, we indeed found the ACC to be implicated in expectancy- and attention- (i.e., target-) related processing. Notably, however, 
greater ACC activity for unexpected compared with expected targets was observed for neutral targets only, suggesting that the top-down mechanisms highlighted in Rolls' (2013) review may not generally apply. In the case of phylogenetic threat, attention mechanisms may bypass or override expectancy-based selection that is based on higher cortical structures in order to achieve more efficient, quick survival responses (cf. LeDoux \& Phelps, 2000).

Mohanty and Sussman (2013) proposed a fronto-parietal attention network consisting of the frontal eye fields, the posterior parietal cortex, and the PCC to be responsible for anticipation-based visual search regarding threat stimuli. These are exactly the areas we found to be implicated in expectancy-related processing of our bird targets (unexpected vs. expected contrasts [Step 3]; but note that Mohanty et al., 2009, in opposition to our own findings, reported these regions to be implicated in cue instead of target processing). What is more, these regions were also among the brain regions whose activity during the expectancy phase (i.e., cue processing) predicted RTs for unexpected versus expected bird targets (Step 4). Yet, these same areas had no predictive power for the detection of unexpected compared with expected spider targets in our study, which might well correspond to the finding of diminished capacity to activate critical neural integration centers (such as the precuneus) when spider phobics expect to encounter spiders (Aue et al., 2015).

Unlike observations outlined in the current paper, Mohanty et al. (2009) reported the fronto-parietal network to be functionally connected with the amygdala and to be characterized by enhanced activity during the anticipation of angry faces, which they interpreted as indicating preferential processing of threat (for similar results, see also Hahn \& Gronlund, 2007; M. A. Williams, Moss, Bradshaw, \& Mattingley, 2005). The difference between the conclusions of the Mohanty study and our own can be related to different factors. First, all of these authors used human faces instead of animals as target stimuli. Despite the fact that both stimulus categories are considered to be a phylogenetic threat, these differences 
might imply that the involved neurocognitive processes differ. Second, the number of items in the visual search task concurrently displayed in the Mohanty et al. (2009) study was considerably lower. Third, although Mohanty et al. (2009) varied anticipation states on a trialto-trial basis, in their case, cues additionally gave spatial information about the to-be-detected targets. Therefore, visual search was considerably restricted, namely, to half of the items in the already small search matrix. In fact, research has shown that increasing the number of items displayed in a search display can considerably change effects observed in visual search studies (e.g., Lipp, Derakshan, Waters, \& Logies, 2004, Experiment 1; Öhman, Flykt, and Esteves, 2001).

The fact that behavioral and neural responses showed similar patterns for both spider phobics and controls (the latter having been characterized by particularly low spider fear) is further consistent with the idea that stimulus saliency in our experiment has not been driven by conscious fear, but by some primitive evolutionary significance (Öhman et al., 2001; Öhman \& Mineka, 2001; Seligman, 1971). Thus, phylogenetic threat detection may, to a certain extent, be independent of expectancy state, and this may hold for different fear levels. Consequently, even if it can be argued that threat-related information processing in phobics is generally abnormal (indeed, we found stronger responding to the spider cues and to the spider targets for phobics compared with controls; see Supplementary Materials, Steps 1 and 2, for details), the current data suggest strong commonalities between phobics and controls in the translation of expectancies into attention deployment.

The asymmetric expectancy effects observed in the current study also need to be interpreted in the context of earlier scientific papers on attention bias, papers that did not consider prior expectancies. For instance, Öhman et al. (2001) postulated different processing modes for phylogenetic threat and neutral targets in visual search tasks. Whereas the processing of fear-relevant stimulus material would be characterized by preattentive parallel 
processing, fear-irrelevant stimulus material would be characterized by postattentive serial processing. Accordingly, brain activity in our study differed massively between the two target types (see Supplementary Materials, Step 2, for details). These neural data complement our earlier observation that the processing of spider targets compared with bird targets in the visual search task is characterized by increased heart and respiration rates (Aue et al., 2016). Together, these findings suggest faster processing and greater processing impact (i.e., response mobilization) of spider compared with bird targets and support the hypothesis of the existence of an innate fear module (Davis \& Lang, 2003; LeDoux \& Phelps, 2000; Öhman \& Mineka, 2001) that facilitates attention orientation to phylogenetic threat stimuli (cf. Yiend, 2010; but see Lipp, 2006, for criticisms regarding this issue).

Our findings may represent a "default" response pattern according to which the detection of threat in the environment is prioritized. This default mode responding possibly relies more strongly on the activation of basic subcortical structures such as the amygdala (e.g., LeDoux \& Phelps, 2000) and somewhat limits the influence of higher cortical structures that exert top-down influences (e.g., those provoked by our cues; see Step 1 analyses in the Supplementary Materials for higher cortical structures associated with cue processing) when fast responding is requested. This may also explain why we did not find any implication of the OFC in expectancy variations while participants attended to threatening targets. Notably though, our comparison of spider and bird targets (Step 2), without simultaneous consideration of expectancy states and other research in the area, has shown the OFC to be critically implicated in the processing of threatening items. These threat-associated neural responses, which are unmodulated by expectancy variations, may be related to the regulation of (phobic) fear (e.g., Hermann et al., 2009) and serve to downsize amygdalar and insular activity (Goldin, McRae, Ramel, \& Gross, 2008). 
It remains to be clarified why phobics and controls displayed oppositely directed correlations between PCC activity for bird versus spider cues in the expectancy phase and RTs for unexpected versus expected bird targets when we tested the predictive power of expectancy-related neural activity for subsequent RTs (Step 4; Figure 4b; negative correlation for phobics, positive correlation for controls). The PCC has been shown to be essential for selective attention (Corbetta \& Shulman, 2002) and suggested to constitute a central hub that relates motivation and top-down control of attention (Small et al., 2005). Along these lines, it has been found that the more the PCC is activated during the stimulus encoding phase, the greater the impact of the respective piece of information on later cognitive processing (e.g., Schiller, Freeman, Mitchell, Uleman, \& Phelps, 2009). The data presented here propose that expectancy-related PCC activity may promote attentional shifting for neutral targets, but differently in phobics and controls. The greater the PCC activity in controls was for bird cues compared with spider cues, the more these participants benefitted from the presentation of the cues, as reflected in their RTs for bird target detection. This result is consistent with Schiller et al.'s (2009) observation of a greater impact of to-be-encoded material on later cognitive processing: Increased PCC activity for bird cues rather than spider cues may correspond with increased processing depth, thus hastening the subsequent detection of birds. Greater PCC activity for spider than for bird cues in phobics, in contrast, may go hand in hand with an increased alarm state, which then, as a by-product, hastens detection of non-threatening bird targets. That these effects in controls and phobics were restricted to the bird targets might again be explained by the existence of specialized fear pathways that preclude such situationspecific variations in the overall attentive state from influencing the detection of threat in the environment.

Finally, it is important to emphasize that the data we present here, for now, are not congruent with the idea of bidirectional influences between different types of cognitive bias in 
fear and anxiety (see Aue \& Okon-Singer, 2015; Hirsch et al., 2006; Peschard, \& Philippot, 2015). However, they do not allow dismissal of the opposite influence, namely, an impact of attention deployment on expectancy formation. They further do not rule out that the influence of one bias on the other is mediated by a third, here unconsidered, bias (e.g., memory bias, interpretation bias; see Aue \& Okon-Singer, 2015, for elaboration). In addition, it is still possible that expectancy influences on attention deployment become more prominent when additional factors are considered. For example, motivation, current goals, and task relevance have been shown to modulate responses to targets (Engelmann \& Pessoa, 2014; LichtensteinVidne, Henik \& Safadi, 2007; Sussman, Jin \& Mohanty, 2016; Vogt, De Houwer, Moors, Van Damme \& Crombez, 2010) and might therefore moderate the impact of expectancies on attention deployment to threat. The influence of these factors should hence be investigated in future research.

\section{Limitations}

We told our participants that, in $90 \%$ of all trials, the cues would correctly predict the targets in the visual search task. In reality, however, cues correctly primed the targets in only $70 \%$ of the trials. This discrepancy resulted from the fact that we aimed to ensure a sufficiently high number of valid samples also for those trials that incorrectly primed the targets. If participants recognized the discrepancy, they may have lowered their trust in the presented cues, thus weakening the intended effects of the cue. Yet, there are important counter-arguments regarding such distrust in the cues. First, participants' behavioral and neural responses to the bird targets was strongly influenced by the presented cues (notably, the same cues could have predicted a bird or a spider target). Second, 70\% (instead of 90\%) correct priming constitutes much higher than chance predictive power, and the participants should have nevertheless experienced an increased likelihood of the cued target being the deviant animal in the search matrix. Third, Entel, Tzelgov, and Bereby-Meyer (2014) 
presented data that show that instructions about proportions of presented stimulus categories that do not match real proportions can suffice to provoke specific effects in participants' behavior.

We might also be criticized because the lack of a correlation with brain activity for spider targets (Step 4) could be due to a somewhat restricted variance in RTs for unexpected versus expected spider targets (compared with unexpected vs. expected bird targets; see Figure 4a, bottom, for details). However, there was still considerable variability, and close consideration of Figure 4a shows that reduced variability cannot explain the lack of statistical significance. There simply appears to be no comparable association as in the case of birds. Equally important, interpretation of Step 4 findings is identical to our conclusions drawn from the RT data and our Step 3 results (which cannot be explained by restricted variance in RTs). The fact that we did not observe any significant difference between unexpected and expected spiders, but that we see, at the same time, meaningful and strong differences between unexpected and expected birds, is therefore supportive of the idea of a resetting attentional system whenever spiders have been presented in the visual search array. Together, these data hence corroborate our hypothesis that the detection of spider targets is more resistant to expectancy influences than is the detection of bird targets.

Despite this, from the present study data, we cannot conclude that phylogenetic threat detection is altogether unaffected by prior expectancies. Our statistical analyses for spiders cannot confirm a null hypothesis, and, as discussed in the previous section, there are in fact studies that show an influence of expectancies on the detection of angry faces (cf. Hahn \& Gronlund, 2007; Mohanty et al., 2009; M. A. Williams, Moss, Bradshaw, \& Mattingley, 2005). Therefore, we do not dismiss the possibility that the inclusion of different stimuli and use of other experimental paradigms can still reveal expectancy influences on attentional deployment to phylogenetic threat. Yet, apart from our own study, existing studies showing 
an influence of prior expectancies on attention to threat did not directly compare effects for neutral versus phylogenetic threat stimuli. Our observation of a strong influence of prior expectancies on the detection of the neutral birds, while the exact same prior expectancies did not significantly impact the detection of spiders, is thus suggestive in that attentional mechanisms for phylogenetic threat might be more difficultly penetrated by top-down processing.

However, these results clearly remain to be replicated with other types of stimuli (e.g., snakes, angry faces) and in other experimental settings. For instance, it is possible that the fact that we informed our participants that we investigated brain responses in response to different animals somehow changed their natural responding. Certainly, these contextual factors may have affected our participants' expectancies and thereby, their detection speed. Yet, for several reasons, we do not think that our study information can completely explain the asymmetric expectancy effects for spider versus bird targets in our study. First, our participants were not recruited to participate in an experiment labeled spider detection. Instead, the study aims specified that we were interested in responses to spiders and birds with no special emphasis on spiders. The appearance of spiders may have altered this balanced situation for the phobic participants; however, it cannot explain the findings in the control group. Second, our participants were also asked about fear of birds in the telephone interview preceding the study so as not to evoke the impression that we were interested only in spiders. Third, prioritized processing of spiders compared with birds has been found in numerous studies not considering prior expectancies, namely, in the area of attention bias. This suggests that different mechanisms do indeed act during the detection of threatening spiders and neutral birds. A resetting attentional system for threat detection (i.e., preattentive processing) may prevent fatal interference by various disturbing external and internal factors, one of these being prior expectancies. 


\section{Summary and Conclusions}

We investigated the idea of attentional mechanisms that are specific to the processing of phylogenetic threat. Concretely, we had hypothesized that attention deployment in early responding to threat (i.e., preattentive processing; e.g., Öhman \& Mineka, 2001) is largely shielded from top-down processes such as prior induced expectancies. Instead, preattentive processing would be characterized by a reset regarding currently subsisting, possibly irrelevant, content. Such a mechanism would ensure that necessary available resources can be fully attributed to existing threat in the environment. Because of their lower survival relevance, no such insulated stimulus processing was predicted for neutral stimuli. Accordingly, we tested whether specific expectancies modulate detection speed for neutral targets but do not modulate it or modulate it less for threatening targets. As predicted, expectancy cues evoked specific behavioral and neural expectancy states and were efficient modulators of the time to detect bird targets. More important, consistent with our hypotheses, there was no impact of expectancy cues on RTs for spider targets - either in the phobics or in the controls. Because the same expectancy cues were used for spiders and birds, the missing effect for spider detections cannot be explained by an inefficient expectancy manipulation. Instead, there must be something special about spider detections.

Our data are in line with the idea of the existence of dedicated fear processing pathways. Such pathways may guarantee the automatic processing of threat stimuli by safeguarding independence from explicit expectancies, thereby ensuring survival. Consequently, human beings will not be hindered in their detection of threat and the preparation of an adaptive, possibly life-saving response- even if they momentarily falsely believe in safety.

Moreover, we were able to show that the asymmetric effect observed in RTs was not simply the result of accelerated motor preparation in the case of unexpected phylogenetic 
threat, but that it must arise much earlier in the processing stream. Otherwise, we would have expected to see more pronounced effects in neural activity when comparing unexpected and expected spider targets; effects that would at least partly overlap with those found for the neutral targets. In sum, the answer to our initial question of whether anticipation can be seen as a propelling force for adaptive attention deployment must, for now, be "no" or "not necessarily." Anticipation may well be a key characteristic of fear as suggested by Aristotle, but it seems that it can determine attention deployment only under specific circumstances, namely, when the number of distractors is low and additional spatial information is provided beforehand, under particular motivational conditions, or when time for responding is sufficiently long. Thus, expectancies are likely not always powerful enough to influence the deployment of attention to threat.

Of note, we have used a similar experimental design in a gambling context in which visual cues predicted gains and losses (Kress, Bristle, \& Aue, 2018). In contrast to our findings in the current study, we did find expectancy influences on attention to loss targets in the visual search display, as well as for gain targets. Therefore, the effects reported in the current paper do not seem to generalize to other types of negative stimuli or to emotional stimuli in general (see Kress \& Aue, 2017, for a detailed description of potential neural mechanisms regarding expectancy-attention interactions in positive affect). Thus, these data, together with the present findings, point to the special character of the processing of phylogenetic threat. Future studies should investigate other types of (phylogenetic) threat stimuli to further determine the degree of specificity of our observations. 


\section{References}

American Psychiatric Association. (2000). Diagnostic and statistical manual of mental disorders (4th ed., text rev.). Washington, DC: Author.

Amir, N., Foa, E. B., \& Coles, M. E. (1998). Automatic activation and strategic avoidance of threat-relevant information in social phobia. Journal of Abnormal Psychology, 107, 285-290.

Aue, T., Chauvigné, L. A. S., Bristle, M., Okon-Singer, H., \& Guex, R. (2016). Expectancy influences on attention to threat are only weak and transient: Behavioral and physiological evidence. Biological Psychology, 121, 173-186.

Aue, T., Guex, R., Chauvigné, L. A. S., \& Okon-Singer, H. (2013). Varying expectancies and the attention bias in phobic and non-phobic individuals. Frontiers in Human Neuroscience, 7, 418.

Aue, T., \& Hoeppli, M. E. (2012). Evidence for an encounter expectancy bias in fear of spiders. Cognition and Emotion, 26, 727-736.

Aue, T., Hoeppli, M. E., Piguet, C., Hofstetter, C., Rieger, S. W., \& Vuilleumier, P. (2015). Brain systems underlying expectancy bias in spider phobia. Cognitive, Affective and Behavioral Neuroscience, 15, 335-348.

Aue T., Hoeppli, M. E., Piguet, C., Sterpenich, V., \& Vuilleumier, P. (2013). Visual avoidance in phobia: Particularities in neural activity, autonomic responding, and cognitive risk evaluations. Frontiers in Human Neuroscience, 7, 194.

Aue, T., Lavelle, L. A., \& Cacioppo, J. T. (2009). Great expectations: What can fMRI tell us about psychological phenomena? International Journal of Psychophysiology, 73, 10-16.

Aue, T., \& Okon-Singer, H. (2015). Expectancy biases in fear and anxiety and their link to biases in attention. Clinical Psychology Review, 42, 83-95.

Bar-Haim, Y, Lamy, D., Pergamin, L., Bakermans-Kranenburg, M. J., \& van IJzendorn, M. H. (2007). Threat-related attentional bias in anxious and nonanxious individuals: A 
meta-analytic study. Psychological Bulletin, 133, 1-24.

Beauchamp, K. G., Kahn, L. E., \& Berkman, E. T. (2016). Does inhibitory control training transfer? Behavioral and neural effects on an untrained emotion regulation task. Social Cognitive and Affective Neuroscience, 11, 1374-1382.

Butler, G., \& Mathews, A. (1983). Cognitive processes in anxiety. Advances in Behaviour Research and Therapy, 5, 51-62.

Cisler, J. M., \& Koster, E. H. W. (2010). Mechanisms of attentional biases towards threat in anxiety disorders: An integrative review. Clinical Psychology Review, 30, 203-216.

Cohen, N., Margulies, D. S., Ashkenazi, S., Schäfer, A., Taubert, M., Henik, A., . . \& \& OkonSinger, H. (2016). Using executive control training to suppress amygdala reactivity to aversive information. Neuroimage, 125, 1022-1031.

Corbetta, M. (1998). Frontoparietal cortical networks for directing attention and the eye to visual locations. Proceedings of the National Academy of Sciences, 95, 831-838.

Corbetta, M., \& Shulman, G. L. (2002). Control of goal-directed and stimulus-driven attention in the brain. Nature Reviews Neuroscience, 3, 201-215.

Dan-Glauser, E. S., \& Scherer, K. R. (2011). The Geneva Affective PicturE Database (GAPED): A new 730 picture database focusing on valence and normative significance. Behavior Research Methods, 43, 468-477.

Davis, M., \& Lang, P. J. (2003). Emotion. In M. Gallagher \& R. J. Nelson (Eds.), Handbook of psychology: Volume 3. Biological psychology (pp. 405-439). New York, NY: Wiley.

Desimone, R., \& Duncan, J. (1995). Neural mechanisms of selective visual attention. Annual Review of Neuroscience, 18, 193-222.

Duncan, J., \& Humphreys, G. (1989). Visual search and stimulus similarity. Psychological Review, 96, 433-458.

Engelmann, J. B., \& Pessoa, L. (2007). Motivation sharpens exogenous spatial attention. Emotion, 7, 668-674. 
Entel, O., Tzelgov, J., \& Bereby-Meyer, Y. (2014). Proportion congruency effects: Instructions may be enough. Frontiers in Psychology, 5, 1108.

Everaert, J., Koster, E. H. W., \& Derakshan, N. (2012). The combined cognitive bias hypothesis in depression. Clinical Psychology Review, 32, 413-424.

Flykt, A., Lindeberg, S., \& Derakshan, N. (2012). Fear makes you stronger: Responding to feared animal targets in visual search. Attention, Perception, and Psychophysics, 74, $1437-1445$

Foa, E. B., \& Kozak, M. J. (1986). Emotional processing of fear: Exposure to corrective information. Psychological Bulletin, 99, 20-35.

Fox, E., Russo, R., Bowles, R., \& Dutton, K. (2001). Do threatening stimuli draw or hold visual attention in subclinical anxiety? Journal of Experimental Psychology: General, 130, 681-700.

Fox, E., Russo, R., \& Dutton, K. (2002). Attentional bias for threat: Evidence for delayed disengagement from emotional faces. Cognition and Emotion, 16, 355-379.

Gauthier, J. B., \& Bouchard, S. (1993). Adaptation canadienne-française de la version révisée du State-Trait Anxiety Inventory de Spielberger. Canadian Journal of Behavioral Sciences, 25, 559-578.

Goldin, P. R., McRae, K., Ramel, W., \& Gross, J. J. (2008). The neural bases of emotion regulation: Reappraisal and suppression of negative emotion. Biological Psychiatry, 63, $577-586$.

Hahn, S., \& Gronlund, S. D. (2007). Top-down guidance in visual search for facial expressions. Psychonomic Bulletin and Review, 14, 159-165.

Hermann, A., Schäfer, A., Walter, B., Stark, R., Vaitl, D., \& Schienle, A. (2009). Emotion regulation in spider phobia: Role of the medial prefrontal cortex. Social Cognitive and Affective Neuroscience, 4, 257-267. 
Hirsch, C. R., Clark, D. M., \& Mathews, A. (2006). Imagery and interpretations in social phobia: Support for the combined cognitive biases hypothesis. Behavior Therapy, 37, $223-236$.

Hutchinson, J. B., \& Turk-Browne, N. B. (2012). Memory-guided attention: Control from multiple memory systems. Trends in Cognitive Sciences, 16, 576-579.

Ingram, R. E. (1984). Toward an information-processing analysis of depression. Cognitive Therapy and Research, 8, 443-477.

Kress, L., \& Aue, T. (2017). The link between optimism bias and attention bias: A neurocognitive perspective. Neuroscience and Biobehavioral Reviews, 80, 688-702.

Kress, L., Bristle, M., \& Aue, T. (2018). Seeing through rose-colored glasses: How optimistic expectancies guide visual attention. Plos One, 13, e0193311.

LeDoux, J. E., \& Phelps, E. A. (2000). Emotional networks in the brain. In M. Lewis \& J. M. Haviland-Jones (Eds.), Handbook of emotions (pp. 157-172). New York: Guilford Press.

Lichtenstein-Vidne, L., Henik, A., \& Safadi, Z. (2007). Task-relevance modulates the effects of peripheral distractors. The Quarterly Journal of Experimental Psychology, 60, 12161226.

Lipp, O. V. (2006). Of snakes and flowers: Does preferential detection of pictures of fearrelevant animals in visual search reflect fear-relevance? Emotion, 6, 296-308.

Lipp, O. V., Derakshan, N., Waters, A. M., \& Logies, S. (2004). Snakes and cats in the flower bed: Fast detection is not specific to pictures of fear-relevant animals. Emotion, 4, 233250.

Mathews, A., Mackintosh, B., \& Fulcher, E. P. (1997). Cognitive biases in anxiety and attention to threat. Trends in Cognitive Sciences, 1, 340-345. 
Mogg, K., \& Bradley, B. P. (1998). A cognitive-motivational analysis of anxiety. Behaviour Research and Therapy, 36, 809-848.

Mogg, K., Bradley, B. P., DeBono, J., \& Painter, M. (1997). Time course of attentional bias for threat information in non-clinical anxiety. Behaviour Research and Therapy, 35, 297-303.

Mohanty, A., Egner, T., Monti, J. M., \& Mesulam, M. M. (2009). Search for a threatening target triggers limbic guidance of spatial attention. Journal of Neuroscience, 29, 1056310572.

Mohanty, A., \& Sussman, T. J. (2013). Top-down modulation of attention by emotion. Frontiers in Human Neuroscience, 7, 102.

Mühlberger, A., Wiedemann, G., Herrmann, M. J., \& Pauli, P. (2006). Phylo- and ontogenetic fears and the expectation of danger: Differences between spider- and flight-phobic subjects in cognitive and physiological responses to disorder-specific stimuli. Journal of Abnormal Psychology, 115, 580-589.

Muris, P., Huijding, J., Mayer, B., den Breejen, E., \& Makkelie, M. (2007). Spider fear and covariation bias in children and adolescents. Behaviour Research and Therapy, 45, 2604-2615.

Öhman, A., Flykt, A., \& Esteves, F. (2001). Emotion drives attention: Detecting the snake in the grass. Journal of Experimental Psychology: General, 130, 466-478.

Öhman, A., \&, Mineka, S. (2001). Fears, phobias, and preparedness: Toward an evolved module of fear and fear learning, Psychological Review, 108, 483-522.

Peschard, V., \& Philippot, P. (2016). Social anxiety and information processing biases: an integrated theoretical perspective. Cognition and Emotion, 30, 762-777.

Rolls, E. T. (2013). A biased activation theory of the cognitive and attentional modulation of emotion. Frontiers in Human Neuroscience, 7, 74. 
Schiller, D., Freeman, J. B., Mitchell, J. P., Uleman, J. S., \& Phelps, E. A. (2009). A neural mechanism of first impressions. Nature Neuroscience, 12, 508-514.

Seligman, M. E. P. (1971). Phobias and preparedness. Behavior Therapy, 2, 307-320.

Small, D. M., Gitelman, D., Simmons, K., Bloise, S. M., Parrish, T., \& Mesulam, M. M. (2005). Monetary incentives enhance processing in brain regions mediating top-down control of attention. Cerebral Cortex, 15, 1855-1865.

Soares, S. C., Esteves, F., Lundqvist, D., \& Öhman, A. (2009). Some animal specific fears are more specific than others: Evidence from attention and emotion measures. Behavioural Research and Therapy, 47, 1032-1042.

Soto, D., Humphreys, G. W., \& Rotshtein, P. (2007). Dissociating the neural mechanisms of memory-based guidance of visual selection. Proceedings of the National Academy of Sciences, 104, 19656.

Spielberger, C. D. (1983). Manual for the State-Trait Anxiety Inventory (Form Y). Palo Alto, CA: Consulting Psychologist Press.

Summerfield, C., Egner, T., Greene, M., Koechlin, E., Mangels, J., \& Hirsch, J. (2006). Predictive codes for forthcoming perception in the frontal cortex. Science, 314, 13111314.

Sussman, T. J., Jin, J., \& Mohanty, A. (2016). Top-down and bottom-up factors in threat-related perception and attention in anxiety. Biological Psychology, 121, 160-172.

Szymanski, J., \& O'Donohue, W. T. (1995). Fear of Spiders Questionnaire. Journal of Behavior Therapy and Experimental Psychiatry, 26, 31-34.

Taylor, S., \& Rachman, S. (1994). Stimulus estimation and the overprediction of fear. British Journal of Clinical Psychology, 33, 173-181.

Vogt, J., De Houwer, J., Moors, A., Van Damme, S., \& Crombez, G. (2010). The automatic orienting of attention to goal-relevant stimuli. Acta Psychologica, 134, 61-69. 
Williams, J. M. G., Watts, F. N., MacLeod, C., \& Mathews, A. (1997). Cognitive psychology and emotional disorders. New York, NY: Wiley.

Williams, M. A., Moss, S. A., Bradshaw, J. L., \& Mattingley, J. B. (2005). Look at me, I'm smiling: Visual search for threatening and nonthreatening facial expressions. Visual Cognition, 12, 29-50.

Wolfe, J. M. (2010). Visual search. Current Biology, 20, R346-R349.

Wolfe, J. M., Butcher, S. J., Lee, C., \& Hyle, M. (2003). Changing your mind: On the contributions of the top-down and bottom-up guidance in visual search for feature singletons. Journal of Experimental Psychology: Human Perception and Performance, $29,483-502$.

World Health Organization. (1992). International statistical classification of diseases and related health problems (10th rev.). Geneva, Switzerland: Author.

World Medical Association. (1999). Proposed revision of the Declaration of Helsinki. British Medical Journal, 147, 18-22.

Yiend, J. (2010). The effects of emotion on attention: A review of attentional processing of emotional information. Cognition and Emotion, 24, 3-47.

Yiend, J., \& Mathews, A. (2001). Anxiety and attention to threatening pictures. Quarterly Journal of Experimental Psychology, 54, 665-681.

Zelano, C., Mohanty, A., \& Gottfried, J. A. (2011). Olfactory predictive codes and stimulus templates in piriform cortex. Neuron, 72, 178-187. 


\section{Acknowledgements}

This research was supported by grant PZ001P1_121590 of the Swiss National Science

Foundation to Tatjana Aue. We kindly thank Bruno Bonet for his support in data acquisition. 


\section{Figure Captions}

Figure 1. Example of experimental trial. Spider $90 \%$, bird $90 \%$, and spider-bird $50 \%$ refer to the expectancy of the target to be a spider or a bird. Only the spider $90 \%$ and the bird $90 \%$ cues are considered in our analyses. Jitters have been introduced to prevent habituation.

Figure 2. Reaction times as a function of Population, Expectancy, and Target. Spider cue and bird cue refer to the spider $90 \%$ and bird $90 \%$ cues, respectively. Error bars depict standard errors. ${ }^{*} p<.001$

Figure 3. Areas displaying differences on viewing unexpected versus expected birds in the target detection phase. $\mathrm{ACC}=$ anterior cingulate cortex; $\mathrm{CB}=$ cerebellum; $\mathrm{CUN}=$ cuneus; IFG = inferior frontal gyrus; IPL = inferior parietal lobule; $\mathrm{MeFG}=$ medial frontal gyrus; $\mathrm{MFG}=$ middle frontal gyrus; $\mathrm{MTG}=$ middle temporal gyrus; $\mathrm{SPL}=$ superior parietal lobule $p<.001 ; k \geq 26$ voxels; spider phobics $(n=20)$ and controls $(n=19)$ combined.

Figure 4. Prediction of reaction times (RTs) for the detection of bird targets (unexpectedexpected) by neural activity (bird cue-spider cue) in the expectancy phase. $p<.001 ; k \geq 26$ voxels. (a) Spider phobics $(n=20)$ and controls $(n=19)$ combined (for comparison reasons, prediction of RTs for spider targets by neural activity in the precuneus is displayed as well; bottom) and (b) differences between spider phobics and controls. PCC $=$ posterior cingulate cortex; PCU $=$ precuneus. ${ }^{*} p<.01 ; * * p>.0005$. 
Table 1

Areas Displaying Differential Activity for Unexpected Versus Expected Birds: Detection

Phase

Peak

MNI Coordinates

Region

$k \quad t$

$\mathrm{x} \quad \mathrm{y} \quad \mathrm{z}$

\section{Unexpected > Expected}

\section{Both Populations (version a)}

$\begin{array}{lccccc}\text { IFG, MFG, INS } & 1002 & 5.84 & 45 & 44 & -14 \\ \text { MeFG, ACC } & 253 & 4.31 & 6 & 41 & 43 \\ \text { IFG } & 44 & 4.29 & -30 & 23 & -8 \\ \text { MFG } & 30 & 3.89 & -39 & 26 & 34 \\ \text { SPL, IPL } & 119 & 3.66 & -27 & -67 & 46 \\ \text { IPL, SPL } & 155 & 4.01 & 48 & -52 & 43 \\ \text { CUN, LING } & 126 & 4.00 & 12 & -85 & 7 \\ \text { CB } & 216 & 4.70 & -9 & -76 & -29 \\ \text { CB } & 48 & 4.03 & 27 & -61 & -32 \\ \text { CB } & 29 & 3.82 & -27 & -67 & -32 \\ \text { CB } & 40 & 4.09 & 0 & -55 & -35\end{array}$

Note. $n=20$ spider phobics and 19 controls. $k=$ cluster size in number of voxels; peak MNI coordinates $=$ Montreal Neurological Institute coordinates referring to the maximally 
activated voxel in each cluster; $\mathrm{ACC}=$ anterior cingulate cortex $\mathrm{CB}=$ cerebellum; $\mathrm{CUN}=$ cuneus; IFG = inferior frontal gyrus; INS = insula; IPL = inferior parietal lobule; $\mathrm{LING}=$ lingual gyrus; $\mathrm{MeFG}=$ medial frontal gyrus; $\mathrm{MFG}=$ middle frontal gyrus; $\mathrm{SPL}=$ superior parietal lobule. Version a refers to the contrast version performed (see text for details).

$p_{\text {uncorr }}<.001 ; k \geq 26$. 
Table 2

Activity in the Expectancy Phase (Bird-Spider) Predicting the Facilitation Effect of the Expectancy Cues on Later Detection of Birds as Seen in $R T$ s ( $\left.R T_{\text {bird unexpected }}-R T_{\text {bird expected }}\right)$

\section{Peak}

MNI Coordinates

Region $\begin{array}{lllllll}k & t & \mathrm{x} & \mathrm{y} & \mathrm{z}\end{array}$

\section{Both Populations}

(version a)

PCU

42

4.06

$-6 \quad-55 \quad 46$

\section{Spider Phobic < Control}

(version b)

PCC

28

4.22

$-18 \quad-61 \quad 13$

Note. $n=20$ spider phobics and 19 controls. $k=$ cluster size in number of voxels; peak MNI

Coordinates $=$ Montreal Neurological Institute coordinates referring to the maximally activated voxel in each cluster; $\mathrm{PCC}=$ posterior cingulate gyrus; $\mathrm{PCU}=$ precuneus. Versions $\mathrm{a}$ and $\mathrm{b}$ refer to the contrast versions performed (see text for details).

$p_{\text {uncorr }}<.001 ; k \geq 26$. 


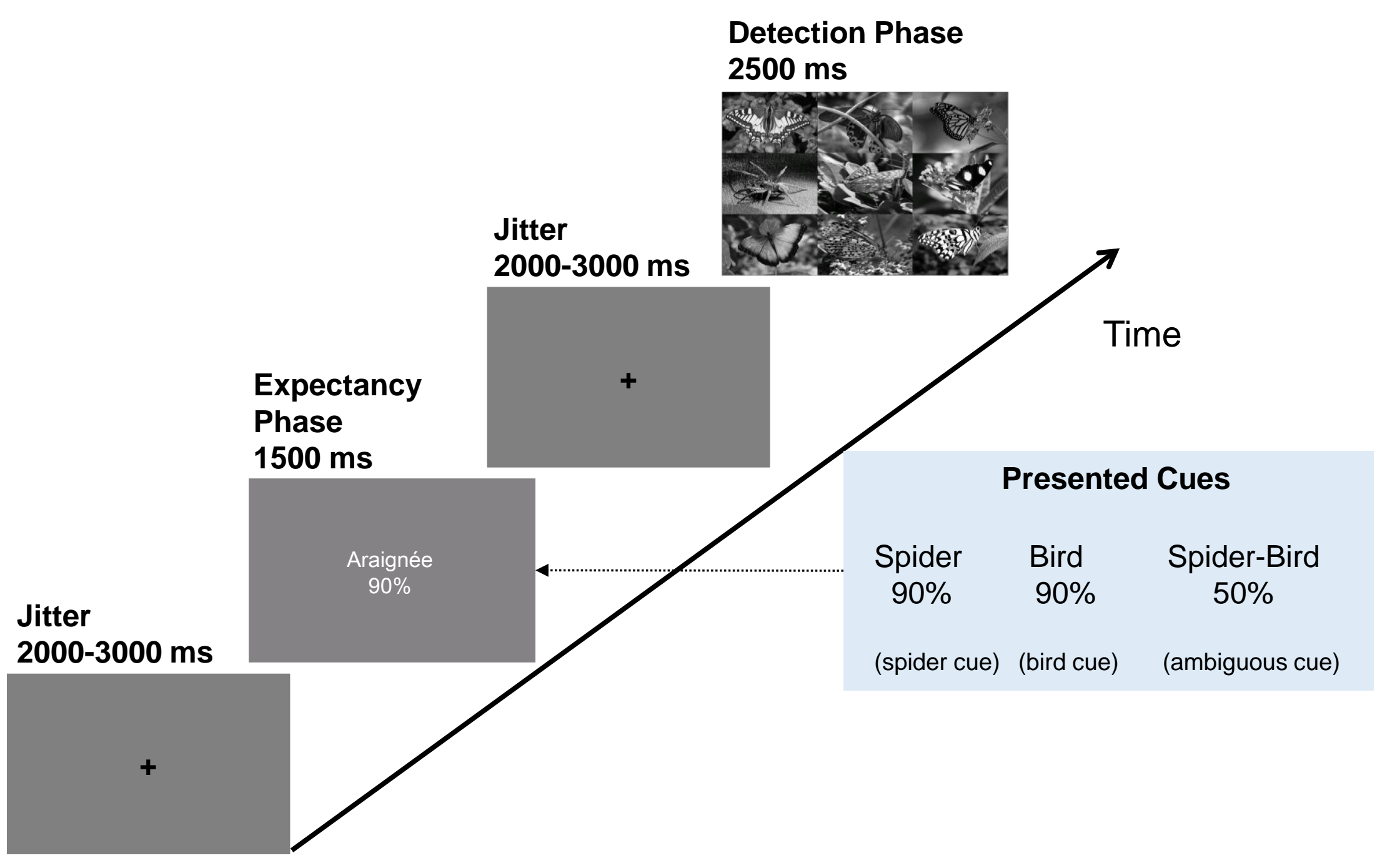




\section{Reaction Times (in ms)}

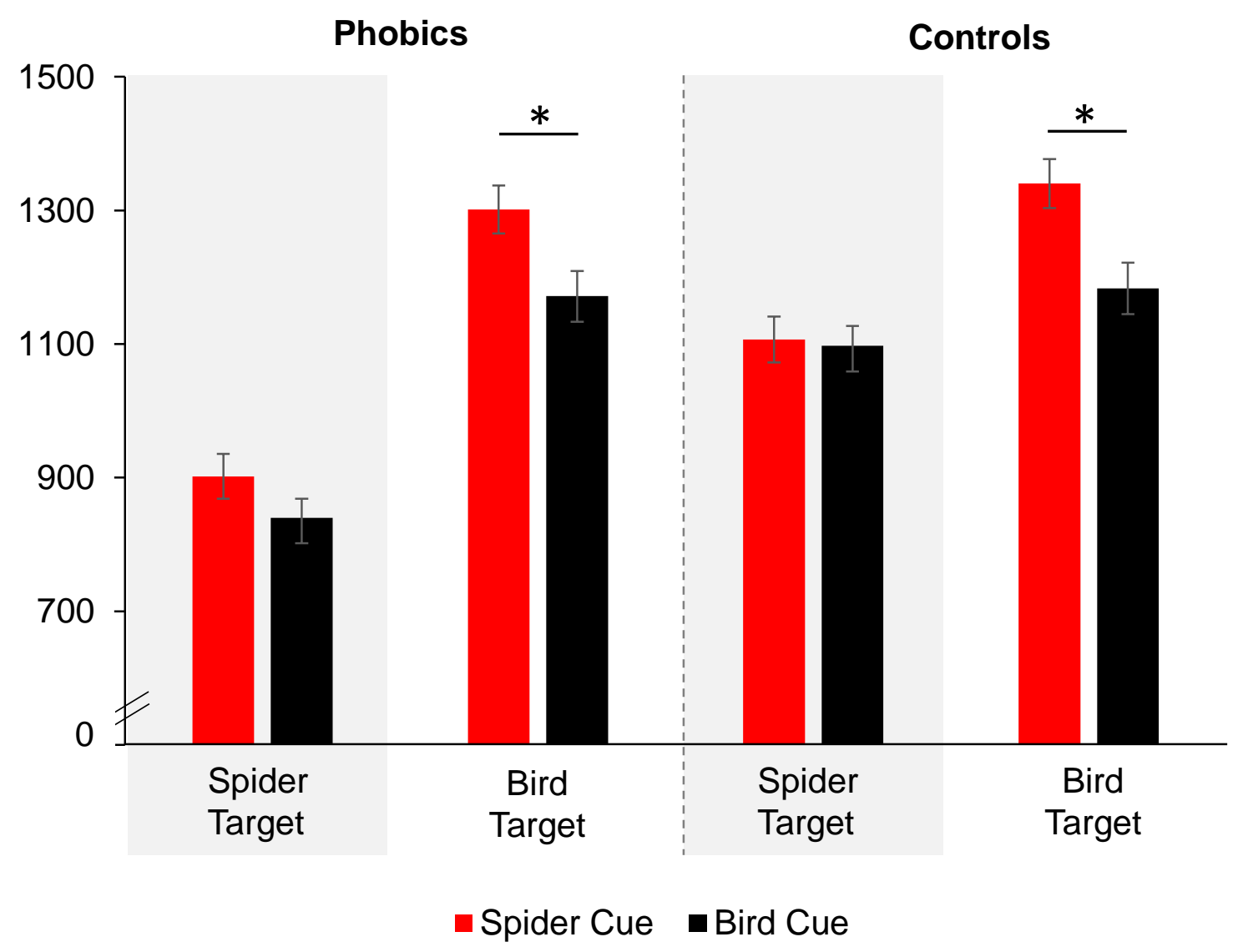


a
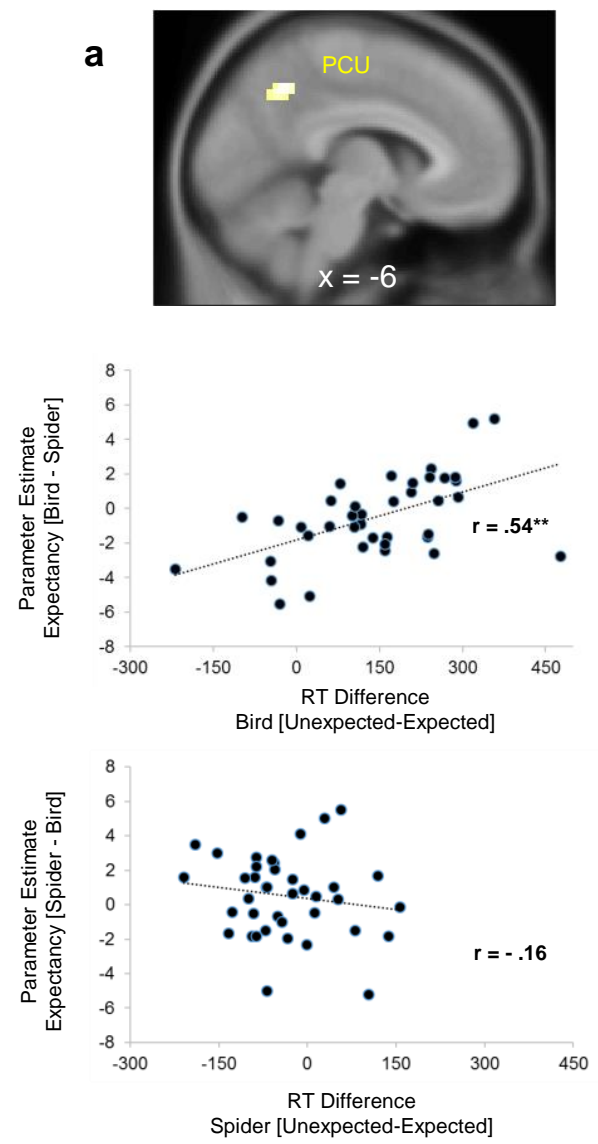

b
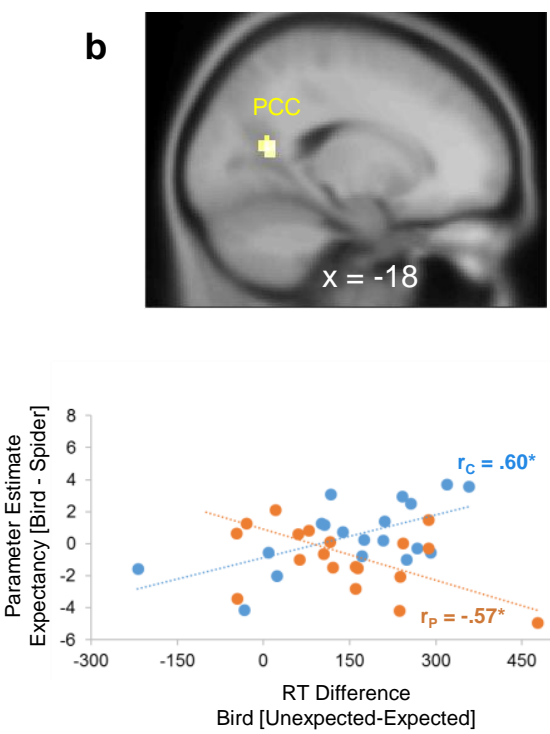

- Phobic Control 


\section{Supplementary Materials}

\section{Additional Analyses Performed}

A. Neural responses upon the presentation of the different cues

(manipulation check, Step 1).

B. Neural responses upon the presentation of the different targets

(manipulation check, Step 2).

C. Differences in neural activity between unexpected and ambiguously-cued targets and between expected and ambiguously-cued targets (addition to Step 3).

C1: Comparison of neural activity for (i) unexpected and ambiguously-cued spiders, and (ii)

expected and ambiguously-cued spiders.

C2: Comparison of neural activity for (i) unexpected and ambiguously-cued birds, and (ii) expected and ambiguously-cued birds. 18 


\section{A. Neural responses on presentation of different cues (manipulation check, Step 1)}

First, we verified whether the induction of expectancies in our participants had been successful. Correspondingly, we performed a whole-brain analysis in which we contrasted responses to spider versus bird cues in the expectancy phase (both spider cue > bird cue and bird cue > spider cue). Two different versions of these contrasts were calculated: (a) for both populations together and (b) for spider phobics versus controls (interaction contrast), permitting the identification of spider phobics' particularities in initial responses toward spider cues (relative to non-phobogenic material). Thus, the latter interaction contrast tested whether the differences revealed by the two contrasts specified above (spider cue $>$ bird cue and bird cue > spider cue, performed on spider targets only) were statistically different between phobics and controls (phobics > controls; note that testing the opposite direction was not necessary this time because the cue contrasts were already tested in both directions). If there was a significant difference, we looked separately into spider phobics (version c) and controls (version d). To avoid alpha inflation, we report only significant clusters containing at least 26 contiguous voxels at $p<.001$. This critical cluster size was calculated by a Monte Carlo simulation with 10,000 iterations, assuming some interdependence between voxels (8mm FWHM), resulting in a corrected whole-brain $p$-value of .001. All parametric maps were rendered on sections of the average $T_{1}$-weighted template brain of all participants.

Combining the two groups of participants (version a) yielded two significant clusters in the visual cortex that were more strongly activated for spider than for bird cues (Figure S1).

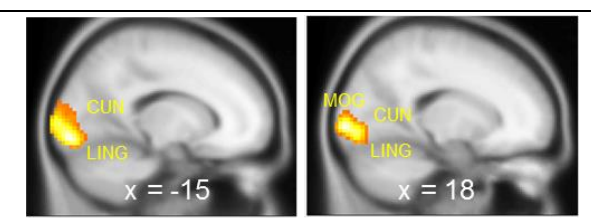

Figure S1. Areas displaying greater activity on viewing spider compared with bird cues in the expectancy phase; version a: spider phobics $(n=20)$ and controls $(n=19)$ combined.

$p<.001 ; k \geq 26$ voxels; spider cue $>$ bird cue. See Table $\mathrm{S} 1$ for definitions of abbreviations. 
When both groups of participants were contrasted with each other (version b),

unsurprisingly, the spider phobics showed more pronounced activity for spider compared with bird cues in various brain areas (Figure S2).
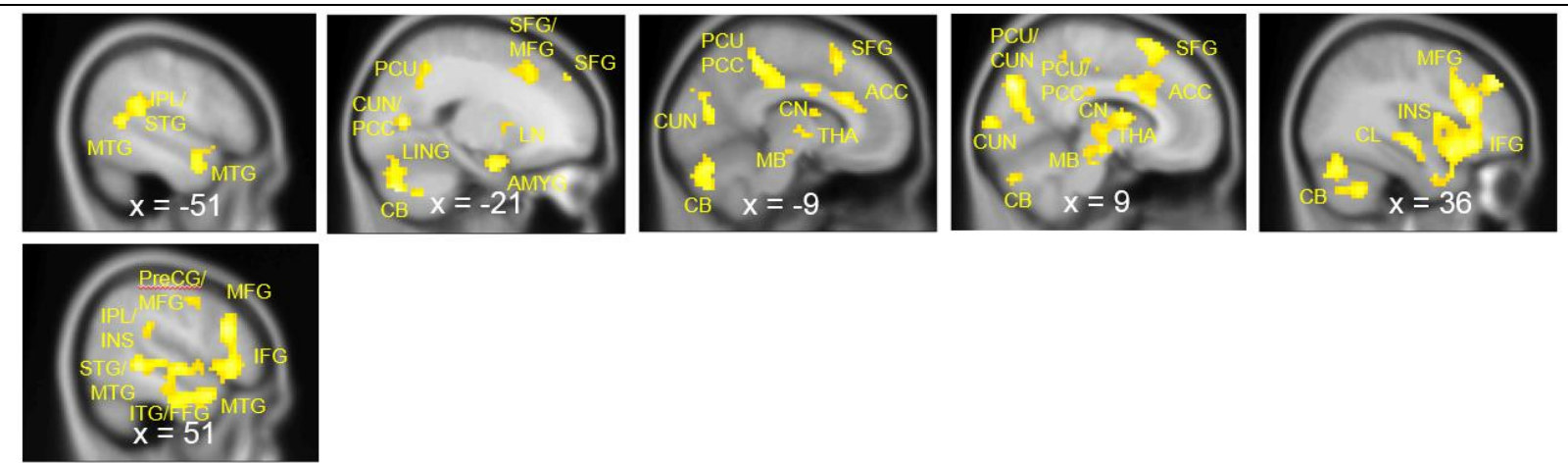

Figure S2. Areas displaying greater activity on viewing spider compared with bird cues in the expectancy phase; version b: spider phobics $(n=20)>$ controls $(n=19)$.

$p<.001 ; k \geq 26$ voxels; spider cue $>$ bird cue. See Table S1 for definitions of abbreviations.

Specifically, in phobics (version c), differential increases for spider versus bird cues were observed in the lateral prefrontal cortex — the dorsal and the ventral part — as well as in the ACC, precuneus, amygdala, insula, and thalamus (see Figure S3 for a contrast in spider phobics only; see Table S1 for completeness). This clearly demonstrates that the induction of expectancies was successful.
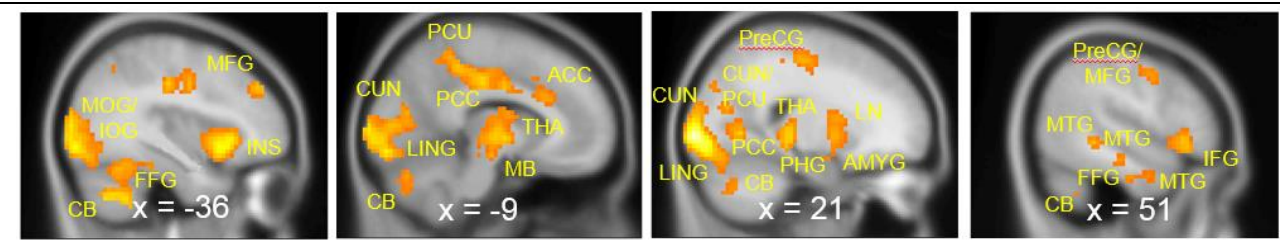

Figure S3. Areas displaying greater activity on viewing spider versus bird cues in the expectancy phase; version c: spider phobics $(n=20)$.

$p<.001 ; k \geq 26$ voxels; spider cue $>$ bird cue. See Table S1 for definitions of abbreviations. 
In controls (version d), activation differences between spider cues and bird cues were not as pronounced. One the one hand, greater activity for spider cues compared with bird cues was limited to visual areas in the lingual gyrus and cuneus; on the other hand, they showed greater activity for bird cues compared with spider cues in a cluster within the inferior and middle frontal gyri (Figure S4).

Figure S4. Areas displaying differences on viewing spider versus bird cues in the expectancy phase;
version d: controls $(\boldsymbol{n}=\mathbf{1 9})$.
$p<.001 ; k \geq 26$ voxels; left: spider cue $>$ bird cue; right: bird cue $>$ spider cue. See Table S1 for definitions of
abbreviations.


Table S1

Areas Displaying Differential Activity for Spider Cues Versus Bird Cues: Expectancy Phase

\begin{tabular}{|c|c|c|c|c|c|}
\hline \multirow[b]{3}{*}{ Region } & \multirow[b]{3}{*}{$k$} & \multirow[b]{3}{*}{$t$} & \multicolumn{3}{|c|}{ Peak } \\
\hline & & & \multicolumn{3}{|c|}{ MNI Coordinates } \\
\hline & & & $\mathrm{x}$ & $\mathrm{y}$ & $\mathrm{z}$ \\
\hline
\end{tabular}

\section{$\underline{\text { Spider Cue }>\text { Bird Cue }}$}

\section{Both Populations (version a)}

MOG, SOG, IOG, CUN, LING, FFG,

CB

LING, CUN, MOG

Spider Phobic $>$ Control (version b)

IFG, INS, MeFG, ACC, MFG, SFG,

STG, MTG, ITG, THA, LN, CN, CL,

AMYG, HIP, PHG, MB, Pons,

PreCG, FFG,

ACC

SFG, MFG

MFG, IFG, PreCG

STG, MTG, PCC, PCU, PCL, IPL,

PoCG, PreCG, MFG, INS

CUN, PCU, PCC

CUN, MOG

PCU

$\mathrm{CN}, \mathrm{LN}$
$966 \quad 8.30$

232

8.12

$\begin{array}{lll}-15 & -91 & -11\end{array}$

$\begin{array}{lll}18 & -97 & -2\end{array}$ $\begin{array}{lllll}4390 & 5.33 & 48 & 11 & -26\end{array}$

$31 \quad 3.92$

$\begin{array}{lll}-9 & -4 & 31\end{array}$

$57 \quad 4.25$

$\begin{array}{lll}-27 & 47 \quad 40\end{array}$

$36 \quad 3.75$

$\begin{array}{lll}42 & -1 & 34\end{array}$

739

5.34

$\begin{array}{lll}-60 & -37 & 16\end{array}$

$454 \quad 5.06$

$\begin{array}{lll}9 & -82 & 34\end{array}$

99

4.22

$\begin{array}{lll}12 & -97 & 7\end{array}$

$36 \quad 3.77$

$\begin{array}{lll}-24 & -55 \quad 46\end{array}$

73

$-12 \quad-1 \quad 16$ 


\section{Spider Phobic (version c)}

CUN, PCC, MOG, LING, FFG, IOG,

IPL, SPL, MTG, PCL, PoCG, PreCG,

MFG, MeFG, ACC, INS, CB

$\begin{array}{rrrrr}4837 & 7.81 & 18 & -97 & -2 \\ 86 & 4.55 & 45 & -13 & -26 \\ 29 & 3.53 & 6 & -43 & 55 \\ 54 & 3.80 & 51 & 2 & 43 \\ 149 & 4.88 & 36 & 47 & 37 \\ 56 & 4.65 & -39 & 41 & 34 \\ 39 & 3.72 & 9 & 17 & 64\end{array}$

SFG, MeFG

IFG, INS, THA, LN, CN, CL,

AMYG, HIP, PHG, HTH, MB, Pons,

STG, MTG, PreCG

Control (version d)

LING, CUN

\section{$\underline{\text { Bird Cue > Spider Cue }}$}

\section{Control (version d)}

IFG, MFG

$109 \quad 4.35$

42

$29 \quad 13$

Note. $n=20$ spider phobics and 19 controls. $k=$ cluster size in number of voxels; peak MNI coordinates $=$ Montreal Neurological Institute coordinates referring to the maximally activated voxel in each cluster; $\mathrm{ACC}=$ anterior cingulate cortex $\mathrm{AMYG}=$ amygdala $\mathrm{CB}=$ cerebellum; $\mathrm{CL}=$ claustrum $; \mathrm{CN}=$ caudate nucleus; $\mathrm{CUN}=$ cuneus $; \mathrm{FFG}=$ fusiform gyrus; 
$\mathrm{HIP}=$ hippocampus; HTH = hypothalamus; IFG = inferior frontal gyrus; INS = insula; IOG $=$ inferior occipital gyrus; IPL = inferior parietal lobule; ITG = inferior temporal gyrus; LING = lingual gyrus; $\mathrm{LN}=$ lentiform nucleus; $\mathrm{MB}=$ midbrain; $\mathrm{MeFG}=$ medial frontal gyrus; $\mathrm{MFG}$ $=$ middle frontal gyrus; $\mathrm{MOG}=$ middle occipital gyrus $; \mathrm{MTG}=$ middle temporal gyrus; $\mathrm{PCC}$ $=$ posterior cingulate cortex $\mathrm{PCL}=$ paracentral lobule $\mathrm{PCU}=$ precuneus $; \mathrm{PHG}=$ parahippocampal gyrus; $\mathrm{PoCG}=$ postcentral gyrus; $\mathrm{PreCG}=$ precentral gyrus; $\mathrm{SFG}=$ superior frontal gyrus; $\mathrm{SOG}=$ superior occipital gyrus; $\mathrm{SPL}=$ superior parietal lobule; $\mathrm{STG}=$ superior temporal gyrus; $\mathrm{THA}=$ thalamus. Versions a-d refer to the contrast versions performed (see text for details).

$p_{\text {uncorr }}<.001 ; k \geq 26$. 


\section{B. Neural responses on presentation of different targets (manipulation check, Step 2)}

Analyses were similar to Step 1 but for spider versus bird targets in the detection phase (i.e., the visual search task). The analysis of both groups combined yielded, first, greater activity for spider than for bird targets within the cingulate cortices (anterior and posterior), the superior frontal gyrus, and parts of the left inferior parietal lobule (Figure S5).
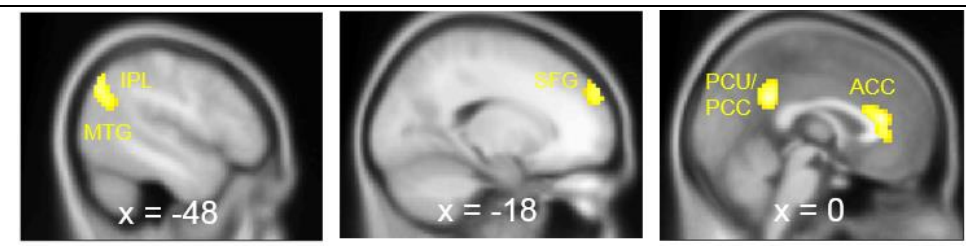

Figure S5. Areas displaying greater activity on viewing spider compared with bird targets in the detection phase; version a: spider phobics $(n=20)$ and controls $(n=19)$ combined.

$p<.001 ; k \geq 26$ voxels; spider target $>$ bird target. See Table $\mathrm{S} 2$ for definitions of abbreviations.

Bird targets rather than spider targets, in contrast, provoked greater activity within the inferior frontal gyrus, various occipital areas, and bilaterally in the superior parietal lobule (extending into the inferior parietal lobule; Figure S6).
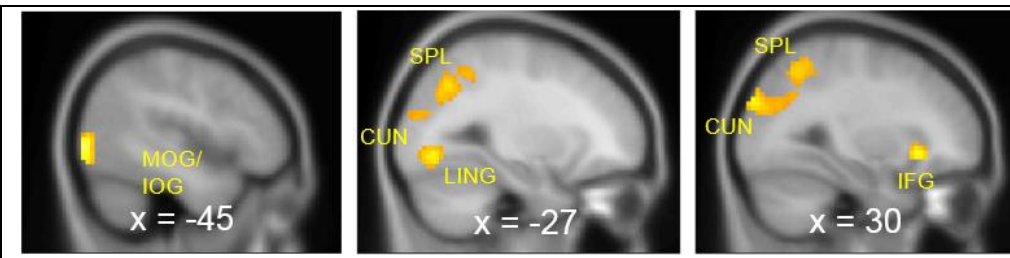

Figure S6. Areas displaying greater activity on viewing bird compared with spider targets in the detection phase; version a: spider phobics $(n=20)$ and controls $(n=19)$ combined.

$p<.001 ; k \geq 26$ voxels; bird target $>$ spider target. See Table S2 for definitions of abbreviations.

When both groups of participants were contrasted with each other (version b; Figure S7; see Table S2 for details), greater activity for spider than for bird targets in spider phobics versus controls was observed in many areas overlapping with those also differentially 
activated during spider expectancy, as described above (cf. Step 1), thus confirming clear group differences regarding the responses to the two targets.
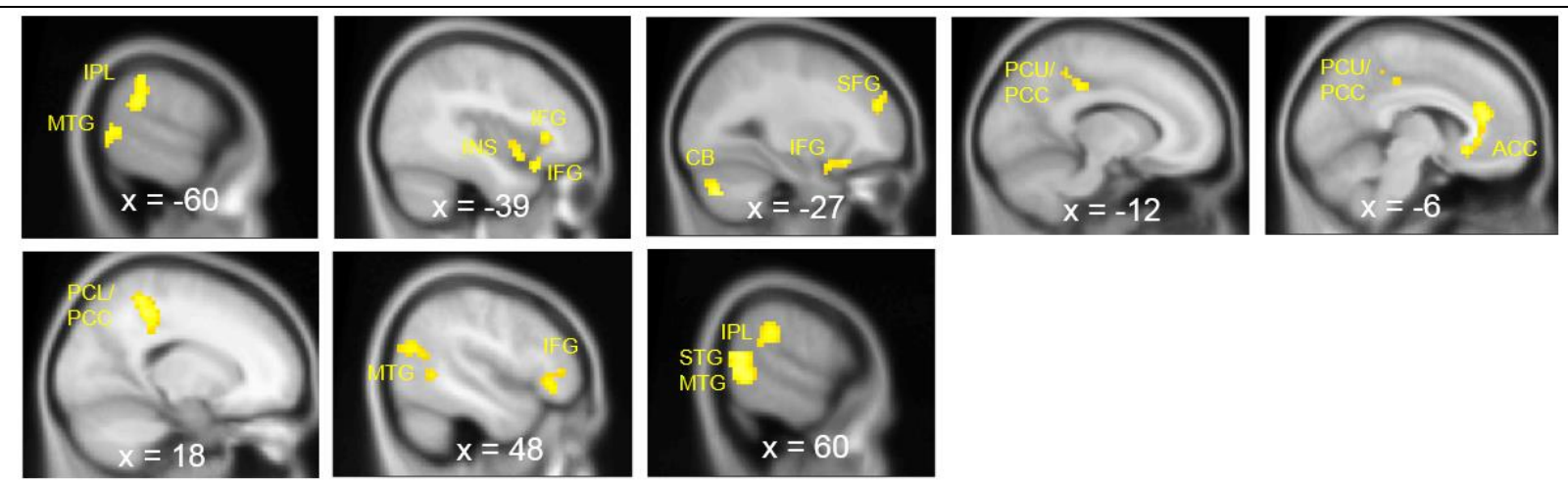

Figure S7. Areas displaying greater activity on viewing spider compared with bird targets in the detection phase; version b: spider phobics $(n=20)>$ controls $(n=19)$. $p<.001 ; k \geq 26$ voxels; spider target $>$ bird target. See Table S2 for definitions of abbreviations.

In spider phobics (version c), areas that were particularly activated during detection of spiders (compared with birds) included, among others, the cingulate cortex (ACC and PCC), the insula, and the lateral prefrontal cortex in the superior and inferior frontal gyri (Figure S8; see Table S2 for completeness).
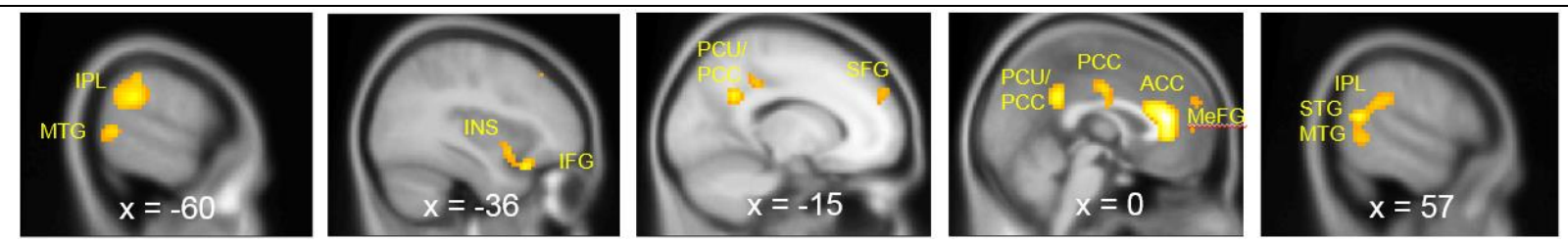

Figure S8. Areas displaying greater activity on viewing spider compared with bird targets in the detection phase; version c: spider phobics $(n=20)$.

$p<.001 ; k \geq 26$ voxels; spider target $>$ bird target. See Table S2 for definitions of abbreviations.

In addition, spider phobics showed lower activity for spider targets than bird targets in two ventral areas within the visual cortex (Figure S9). 

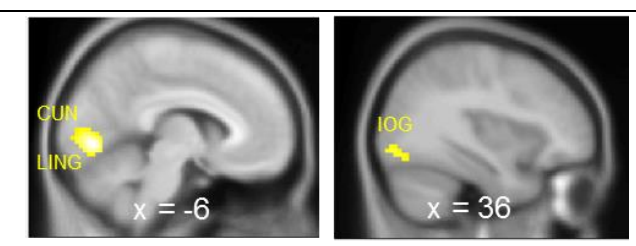

Figure S9. Areas displaying greater activity on viewing bird compared with spider targets in the detection phase; version c: spider phobics $(n=20)$.

$p<.001 ; k \geq 26$ voxels; bird target $>$ spider target. See Table $\mathrm{S} 2$ for definitions of abbreviations.

The control group (version d) did not display significantly greater activity for spider than for bird targets in any brain area. By contrast, these participants were characterized by increased activity for bird rather than spider targets within the inferior frontal gyrus, insula, middle frontal gyrus, inferior parietal cortex, and various occipital areas (Figure S10).

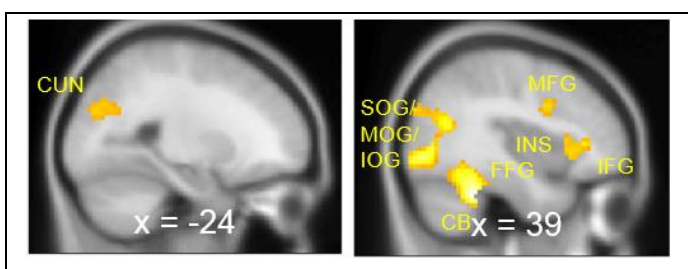

Figure S10. Areas displaying greater activity on viewing bird compared with spider targets in the detection phase; controls $(n=19)$.

$p<.001 ; k \geq 26$ voxels; bird target $>$ spider target. See Table S2 for definitions of abbreviations. 
Table S2

Areas Displaying Differential Activity for Spider Targets Versus Bird Targets: Detection

Phase

Peak

MNI Coordinates

Region

$k \quad t$

$\mathrm{x} \quad \mathrm{y} \quad \mathrm{z}$

\section{$\underline{\text { Spider Target }>\text { Bird Target }}$}

Both Populations (version a)

ACC, MeFG, SFG

22

5.30

$\begin{array}{lll}0 & 32 & 13\end{array}$

SFG

$54 \quad 4.45$

$\begin{array}{lll}-18 & 56 \quad 31\end{array}$

PCC, PCU

249

5.56

$\begin{array}{lll}-6 & -49 & 31\end{array}$

IPL, MTG, STG

$156 \quad 4.66$

$\begin{array}{lll}-48 & -70 \quad 34\end{array}$

Spider Phobic > Control (version b)

$\begin{array}{lccccc}\text { ACC } & 222 & 5.58 & 0 & 35 & 13 \\ \text { IFG } & 28 & 3.94 & -42 & 29 & 1 \\ \text { IFG, MFG } & 92 & 4.31 & 39 & 32 & 4 \\ \text { IFG, STG, INS, MFG } & 109 & 4.15 & -36 & 20 & -20 \\ \text { MTG, STG } & 139 & 4.76 & -51 & -61 & 10 \\ \text { MTG, STG } & 298 & 4.69 & 57 & -55 & 13 \\ \text { SFG, MFG } & 35 & 3.93 & -27 & 47 & 25 \\ \text { PCC, PCU } & 49 & 3.86 & -12 & -34 & 40 \\ \text { PCL, PCC, PCU } & 128 & 4.27 & 18 & -43 & 55 \\ \text { IPL } & 111 & 4.83 & 66 & -31 & 31 \\ \text { IPL } & 99 & 4.26 & -57 & -43 & 28\end{array}$


Spider Phobic (version c)

$\begin{array}{lccccc}\text { ACC, MeFG, SFG, SCG } & 486 & 6.69 & 0 & 32 & 13 \\ \text { SFG, MFG } & 82 & 4.82 & -21 & 56 & 31 \\ \text { SFG, MFG } & 36 & 4.26 & -24 & 44 & 46 \\ \text { IFG, MFG } & 44 & 4.19 & 48 & 35 & -11 \\ \text { IFG, STG, INS } & 168 & 4.44 & -36 & 20 & -20 \\ \text { STG, IFG } & 35 & 3.92 & 36 & 11 & -17 \\ \text { PCC } & 51 & 4.20 & -3 & -13 & 34 \\ \text { PCC, PCU } & 291 & 5.07 & -6 & -49 & 28 \\ \text { IPL, STG, MTG, PoCG } & 266 & 5.51 & 66 & -31 & 31 \\ \text { IPL } & 170 & 4.60 & -60 & -40 & 28 \\ \text { MTG, STG, IPL } & 182 & 4.65 & -51 & -58 & 10 \\ \text { CB } & 45 & 4.93 & -24 & -76 & -38\end{array}$

\section{$\underline{\text { Bird Target }>\text { Spider Target }}$}

\section{Both Populations (version a)}

IFG, INS

$41 \quad 4.76$

$\begin{array}{lll}30 & 23 & -5\end{array}$

MFG, IFG

$242 \quad 5.06$

$39 \quad 29 \quad 22$

CUN, LING, IOG, MOG, SOG, PCU,

PCC, FFG, CB

$1466 \quad 6.63$

$\begin{array}{lll}-6 & -79 & -5\end{array}$

MOG, IOG, FFG, ITG, MTG, CB

335

6.18

$\begin{array}{lll}45 & -79 & -2\end{array}$

IPL, SPL, PoCG

$197 \quad 4.45$

$42 \quad-46 \quad 43$

SPL, IPL

$83 \quad 4.10$

$\begin{array}{lll}-27 & -64 \quad 40\end{array}$

Spider Phobic (version c)

CUN, LING, IOG 


\section{Control (version d)}

IFG, INS

IFG, MFG

MTG, ITG, STG, MOG, IOG, SOG,

PCU, CUN

PCU

PCU

MOG, IOG, FFG, MTG, CB

FFG, CB

LING, CUN, CB
307

67

570

54

31

380

192

379
4.68

3.90

6.42

3.75

3.68

5.67

6.22

4.20
$27 \quad 26 \quad-5$

$\begin{array}{lll}42 & 8 & 28\end{array}$

$\begin{array}{lll}48 & -76 & 1\end{array}$

$\begin{array}{lll}-24 & -79 & 28\end{array}$

$\begin{array}{lll}24 & -58 & 52\end{array}$

$\begin{array}{lll}-39 & -43 & -20\end{array}$

$\begin{array}{lll}39 & -46 & -32\end{array}$

$\begin{array}{lll}-3 & -61 & -38\end{array}$

Note. $n=20$ spider phobics and 19 controls. $k=$ cluster size in number of voxels; MNI coordinates $=$ Montreal Neurological Institute coordinates referring to the maximally activated voxel $(\mathrm{s})$ in each cluster. $\mathrm{ACC}=$ anterior cingulate cortex; $\mathrm{CB}=$ cerebellum; $\mathrm{CUN}=$ cuneus; FFG = fusiform gyrus; IFG = inferior frontal gyrus; INS = insula; $\mathrm{IOG}=$ inferior occipital gyrus; IPL = inferior parietal lobule; $\mathrm{ITG}=$ inferior temporal gyrus; $\mathrm{LING}=$ lingual gyrus $; \mathrm{MeFG}=$ medial frontal gyrus $; \mathrm{MFG}=$ middle frontal gyrus $; \mathrm{MOG}=$ middle occipital gyrus $; \mathrm{MTG}=$ middle temporal gyrus $; \mathrm{PCC}=$ posterior cingulate cortex $; \mathrm{PCL}=$ paracentral lobule; $\mathrm{PCU}=$ precuneus $; \mathrm{PoCG}=$ postcentral gyrus; $\mathrm{SCG}=$ subcallosal gyrus; $\mathrm{SFG}=$ superior frontal gyrus; $\mathrm{SOG}=$ superior occipital gyrus; $\mathrm{SPL}=$ superior parietal lobule; $\mathrm{STG}=$ superior temporal gyrus. Versions a-d refer to the contrast versions performed (see text for details).

$p_{\text {uncorr }}<.001 ; k \geq 26$. 


\section{Differences in neural activity between unexpected and ambiguously cued targets and}

\section{between expected and ambiguously cued targets (addition to Step 3)}

The nature of ambiguously cued trials tremendously differed from that of unambiguously cued trials in our study. For instance, the majority of participants mentioned after the study that they had not trusted ambiguous cues (see Method section for details). For the sake of completeness, however, we also contrasted responses to ambiguously cued spiders and birds with responses to unambiguously cued spiders and birds. Specifically, we performed a wholebrain analysis in which we first contrasted responses to spider targets that were preceded by bird cues (i.e., unexpected spiders), relative to responses to spider targets that were preceded by ambiguous cues (both bird cue > ambiguous cue and ambiguous cue > bird cue; performed on spider targets only). Two different versions of these contrasts were calculated: (a) for both populations together and (b) for spider phobics versus controls (interaction contrast), permitting the identification of spider phobics' particularities in initial responses toward incongruently versus ambiguously cued spider targets. Thus, the latter interaction contrast tested whether the differences revealed by the two contrasts specified above (bird cue > ambiguous cue and ambiguous cue > bird cue, performed on spider targets only) were statistically different between phobics and controls (phobics > controls; note that testing the opposite direction was not necessary because the cue contrasts were already tested in both directions). If there was a significant difference, its nature was identified by looking separately into phobics and controls. The same types of contrasts were performed for the difference between expected and unambiguously cued spider targets and also for the difference between unexpected and ambiguously cued bird targets, as well as for the difference between expected and ambiguously cued bird targets.

To avoid alpha inflation, we report only significant clusters containing at least 26 contiguous voxels at $p<.001$. This critical cluster size was calculated by a Monte Carlo simulation with 10,000 iterations, assuming some interdependence between voxels (8-mm 
FWHM), resulting in a corrected whole-brain $p$-value of .001. All parametric maps were rendered on sections of the average $T_{1}$-weighted template brain of all participants.

\section{C1: Comparison of neural activity for (i) unexpected and ambiguously cued spiders and (ii)}

\section{expected and ambiguously cued spiders}

(i) When comparing neural activity for those spider targets that had been preceded by bird cues (i.e., unexpected spider targets) with activity for spider targets that had been preceded by ambiguous cues, we found increased activity in the visual cortex (Figure S11; see Table S3 for details) for the unexpected spider targets, but only when spider phobics and controls were combined (version a). Phobics and controls did not differ with respect to their responses to unexpected compared with ambiguously cued spider targets (version b).

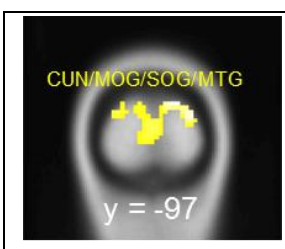

Figure S11. Areas displaying greater activity on viewing unexpected compared with ambiguously cued spider targets in the detection phase; version a: spider phobics $(n=20)$ and controls $(n=19)$ combined. $p<.001 ; k \geq 26$ voxels; unexpected spider target $>$ ambiguously cued spider target. See Table S3 for definitions of abbreviations.

(ii) A highly similar picture emerged when we compared neural activity for those spider targets that had been preceded by spider cues (i.e., expected spider targets) with activity for spider targets that had been preceded by ambiguous cues. Specifically, we observed increased activity in the visual cortex (Figure S12) for the expected spider targets, again only when spider phobics and controls were combined (version a). Phobics and controls did not differ with respect to their responses to expected compared with ambiguously cued spider targets (version b). 


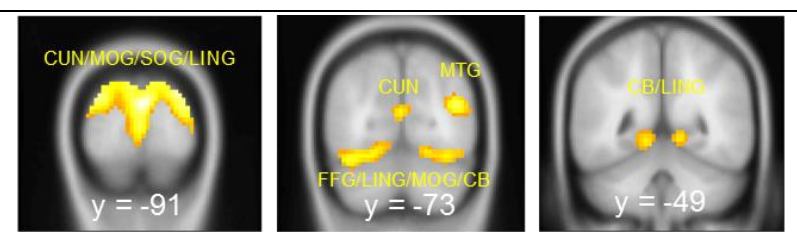

Figure S12. Areas displaying greater activity on viewing expected compared with ambiguously cued spider targets in the detection phase; version a: spider phobics $(n=20)$ and controls $(n=19)$ combined.

$p<.001 ; k \geq 26$ voxels; expected spider target $>$ ambiguously cued spider target. See Table S3 for definitions of abbreviations.

Together, therefore, our analyses for the ambiguous spider targets revealed highly similar neural differences between both types of unambiguously cued spider target trials, on the one hand, and ambiguously cued spider target trials, on the other hand. These differences are located in the visual cortex, suggesting stronger visual engagement in the unambiguously cued trials, which possibly relates to the fact that the ambiguous cues provoked overall greater confusion and complicated visual search activities. 


\section{Table S3}

Areas Displaying Differential Activity for (i) Unexpected Versus Ambiguously Cued Spiders and (ii) Expected Versus Ambiguously Cued Spiders: Detection Phase

Peak

MNI Coordinates

Region

$k$

$t$

$\begin{array}{lll}\mathrm{x} & \mathrm{y} & \mathrm{Z}\end{array}$

\section{(i) Unexpected Spider Target > Ambiguously Cued Spider Target}

Both Populations (version a)

CUN, MOG, SOG, MTG

418

4.99

$15 \quad-97$

25

\section{(ii) Expected Spider Target $>$ Ambiguously Cued Spider Target}

\section{Both Populations (version a)}

CUN, MOG, SOG, IOG, LING, FFG,

PCU, MTG, CB

1748

6.64

$\begin{array}{lll}3 & -91 & 22\end{array}$

FFG, LING, MOG, IOG, PHG, CB

$295 \quad 5.08$

$\begin{array}{lll}30 & -79 & -17\end{array}$

CB, PHG, LING

63

4.31

$\begin{array}{lll}-12 & -49 & -5\end{array}$

Note. $n=20$ spider phobics and 19 controls. $k=$ cluster size in number of voxels; MNI

coordinates $=$ Montreal Neurological Institute coordinates referring to the maximally activated voxel(s) in each cluster; $\mathrm{CB}=$ cerebellum; $\mathrm{CUN}=$ cuneus; $\mathrm{FFG}=$ fusiform gyrus; $\mathrm{IOG}=$ inferior occipital gyrus; $\mathrm{LING}=$ lingual gyrus; $\mathrm{MOG}=$ middle occipital gyrus; $\mathrm{MTG}=$ middle temporal gyrus; $\mathrm{PCU}=$ precuneus $; \mathrm{PHG}=$ parahippocampal gyrus; $\mathrm{SOG}=$ superior occipital gyrus. Versions a-d refer to the contrast versions performed (see text for details).

$p_{\text {uncorr }}<.001 ; k \geq 26$. 
expected and ambiguously cued birds

(i) When comparing neural activity in the overall sample (version a) for those bird targets that had been preceded by spider cues (i.e., unexpected spider targets) with activity for bird targets that had been preceded by ambiguous cues, we also found increased activity in the visual cortex (Figure S13; see Table S4 for details) for the unexpected bird targets. However, this time, we additionally observed massively increased activity in various frontal and parietal areas for the unexpected bird targets; areas that strongly overlapped with those arising in the contrast of unexpected versus expected birds.

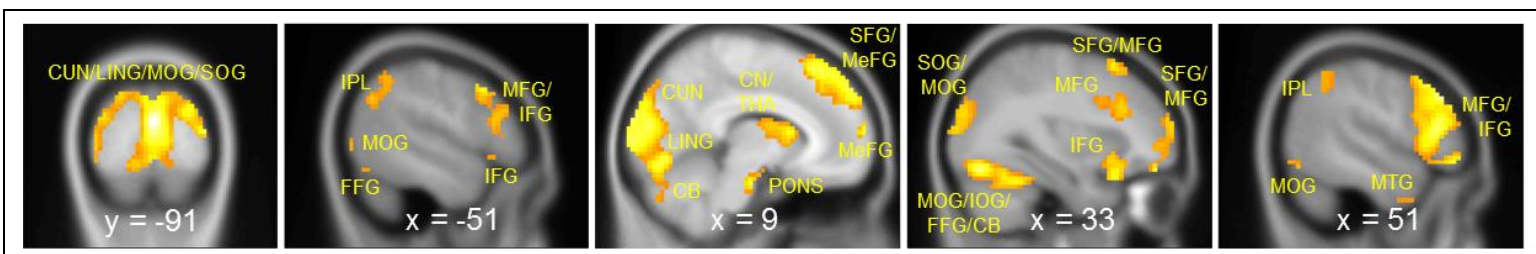

Figure S13. Areas displaying greater activity on viewing unexpected compared with ambiguously cued bird targets in the detection phase; version a: spider phobics $(n=20)$ and controls $(n=19)$ combined. $p<.001 ; k \geq 26$ voxels; unexpected bird target $>$ ambiguously cued bird target. See Table S4 for definitions of abbreviations.

Furthermore, phobics and controls did differ with respect to their responses to unexpected compared with ambiguously cued bird targets (version b). Specifically, phobics were characterized by a stronger distinction of unexpected and ambiguously cued bird target trials as revealed by neural activity within the inferior parietal lobule (Figure S14). As a consequence, we performed contrasts separately for spider phobics and controls. 


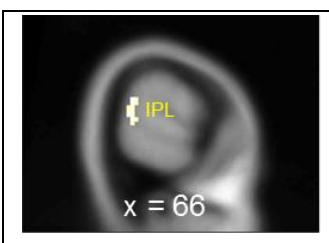

Figure S14. Areas displaying greater activity on viewing unexpected compared with ambiguously cued bird targets in the detection phase; version b: spider phobics $(n=20)>$ controls $(n=19)$.

$p<.001 ; k \geq 26$ voxels; unexpected bird target $>$ ambiguously cued bird target. See Table S4 for definitions of abbreviations.

Differences for the phobic group (version c; Figure 15) strongly resembled those uncovered for the overall sample of participants.
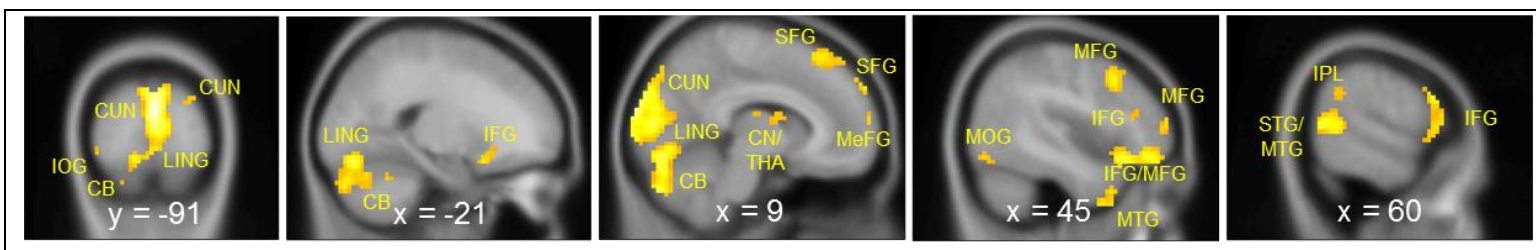

Figure S15. Areas displaying greater activity on viewing unexpected compared with ambiguously cued bird targets in the detection phase; version c: spider phobics $(n=20)$.

$p<.001 ; k \geq 26$ voxels; unexpected bird target $>$ ambiguously cued bird target. See Table S4 for definitions of abbreviations.

Differences for the control group (version d; Figure S16) were highly similar but appeared, on the whole, to be more restricted. Note, however, that the only significant group difference arose in the inferior parietal lobule (see version $b$ above).
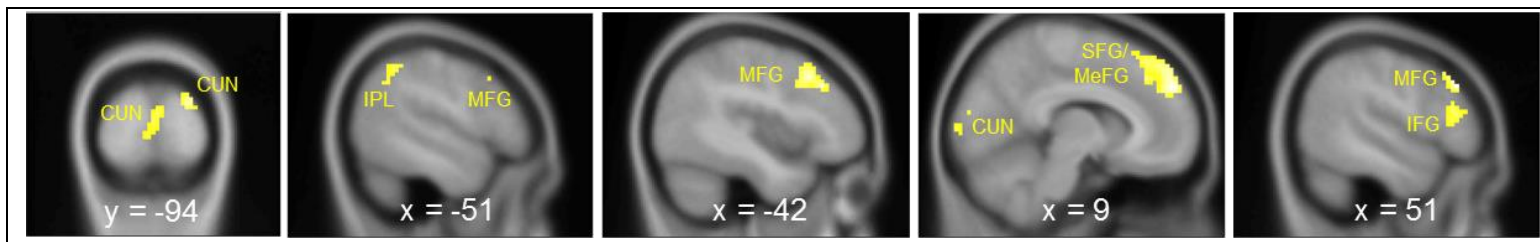

Figure S16. Areas displaying greater activity on viewing unexpected compared with ambiguously cued bird targets in the detection phase; version d: controls $(n=19)$.

$p<.001 ; k \geq 26$ voxels; unexpected bird target $>$ ambiguously cued bird target. See Table S4 for definitions of abbreviations. 
(ii) Finally, the picture that emerged, when we compared neural activity for those bird targets that had been preceded by bird cues (i.e., expected bird targets) with activity for bird targets that had been preceded by ambiguous cues, strongly resembled all other contrasts performed with respect to the ambiguous cues. Specifically, we observed increased activity in the visual cortex (Figure S17) for the expected bird targets, again only when spider phobics and controls were combined (version a). Phobics and controls did not differ with respect to their responses to expected compared with ambiguously cued bird targets (version b).

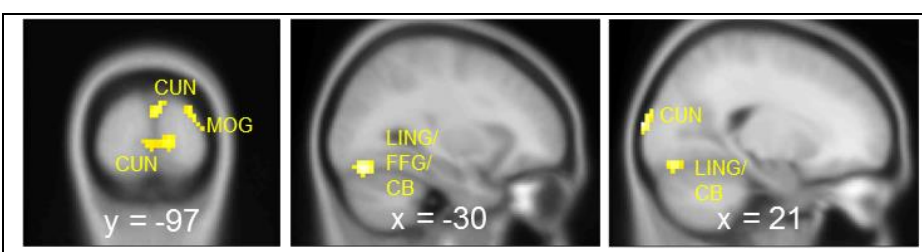

Figure S17. Areas displaying greater activity on viewing expected compared with ambiguously cued bird targets in the detection phase; version a: spider phobics $(n=20)$ and controls $(n=19)$ combined.

$p<.001 ; k \geq 26$ voxels; expected bird target $>$ ambiguously cued bird target. See Table S4 for definitions of abbreviations.

Hence, our data suggest that, irrespective of the nature of the target, differences between ambiguously and unambiguously cued trials are restricted to the visual cortex, which might be explained by a complicated visual search following the less predictive ambiguous cues. Only in the case of bird targets do we see an additional implication of various frontal and parietal areas, which further strengthens our hypothesis that expectancy influences on attention to threat are more restricted. 
Table S4

Areas Displaying Differential Activity for (i) Unexpected Versus Ambiguously Cued Birds and (ii) Expected Versus Ambiguously Cued Birds: Detection Phase

Peak

MNI Coordinates

Region

$k \quad t$

$\begin{array}{lll}X & Y & Z\end{array}$

\section{(i) Unexpected Bird Target $>$ Ambiguously Cued Bird Target}

Both Populations (version a)

MFG, MeFG, IFG, SFG, STG, PreCG

$2346 \quad 6.01$

$\begin{array}{lll}51 & 32 & 34\end{array}$

MFG, IFG, SFG

351

5.16

$\begin{array}{lll}-45 & 20 & 40\end{array}$

MFG

41

4.16

$\begin{array}{lll}-39 & 53 & -2\end{array}$

IFG, STG

70

4.55

$\begin{array}{lll}-21 & 14 & -17\end{array}$

INS, MTG

58

4.65

$\begin{array}{lll}42 & 14 & -47\end{array}$

PONS, PHG

44

5.15

$\begin{array}{lll}9 & -16 & -26\end{array}$

CN, THA

155

4.91

$9 \quad 11 \quad 10$

IPL

$166 \quad 4.37$

$\begin{array}{lll}-54 & -64 & 31\end{array}$

IPL

$81 \quad 4.02$

$45 \quad-46 \quad 52$

CUN, LING, FFG, MOG, IOG, SOG,

PCU, CB

Spider Phobic > Control (version b)

IPL, STG

Spider Phobic (version c)

MFG, SFG

IFG, MFG, STG

$708 \quad 5.01$

$\begin{array}{lll}42 & 23 & -17\end{array}$ 
IFG

SFG

STG, MTG, IPL

ITG, MTG

THA, CN

CUN, LING, FFG, MOG, IOG, PCU,

CB

CB

CB

\section{Control (version d)}

MFG, IFG, SFG

MFG, IFG

SFG, MFG

IPL

PHG, CUN, SOG

CUN
$130 \quad 4.75$

$37 \quad 3.98$

$26 \quad 3.60$

1982

6.30

$\begin{array}{lll}3 & -94 & 19\end{array}$

$28 \quad 4.09$

32

4.00

$\begin{array}{lll}-45 & -64 & -38\end{array}$

$21 \quad-40 \quad-50$ $\begin{array}{lll}45 & 8 & -47\end{array}$

$\begin{array}{lll}9 & -1 & 13\end{array}$

$\begin{array}{lll}-21 & 14 & -17\end{array}$

$\begin{array}{lll}9 & 29 & 55\end{array}$

$\begin{array}{lll}60 & -58 & 10\end{array}$

9

8


activated voxel(s) in each cluster; $\mathrm{CB}=$ cerebellum; $\mathrm{CN}=$ caudate nucleus; $\mathrm{CUN}=$ cuneus; $\mathrm{FFG}=$ fusiform gyrus; IFG = inferior frontal gyrus; INS = insula; IOG = inferior occipital gyrus; IPL = inferior parietal lobule; ITG = inferior temporal gyrus; LING = lingual gyrus; $\mathrm{MeFG}=$ medial frontal gyrus; $\mathrm{MFG}=$ middle frontal gyrus; $\mathrm{MOG}=$ middle occipital gyrus; $\mathrm{MTG}=$ middle temporal gyrus $; \mathrm{PCU}=$ precuneus $; \mathrm{PHG}=$ parahippocampal gyrus; PreCG = precentral gyrus; SFG = superior frontal gyrus; SOG = superior occipital gyrus; $\mathrm{STG}=$ superior temporal gyrus; THA = thalamus. Versions a-d refer to the contrast versions performed (see text for details).

$p_{\text {uncorr }}<.001 ; k \geq 26$. 